\title{
ON THE PROOF OF THE SOLVABILITY OF A LINEAR PROBLEM ARISING IN MAGNETOHYDRODYNAMICS WITH THE METHOD OF INTEGRAL EQUATIONS
}

\author{
SH. SAHAEV AND V. A. SOLONNIKOV \\ Dedicated to Professor G. Grubb \\ on the occasion of her jubilee
}

\begin{abstract}
The paper is concerned with a linear system of Fredholm-Volterra singular integral equations arising in the study of a linearized initial-boundary value problem of magnetohydrodymnamics for a fluid surrounded by an infinite vacuum region. It is proved that this system is solvable in the class of continuous functions satisfying the Hölder condition with respect to the spatial variables, which yields a classical solution of the problem in question.
\end{abstract}

\section{§1. Statement of the PRoblem AND MAin Result}

Let $\Omega_{1}$ be a bounded simply connected domain with boundary $S$ of class $C^{1+\alpha}, \alpha \in$ $(0,1)$, and let $\Omega_{2}=\mathbb{R}^{3} \backslash \bar{\Omega}_{1}$. In $\Omega_{1} \cup \Omega_{2}$, we consider the following initial-boundary value problem for the vector field $\boldsymbol{H}(x, t), x \in \Omega_{1} \cup \Omega_{2}$ :

$$
\left\{\begin{array}{l}
\mu_{1} \boldsymbol{H}_{t}(x, t)+\alpha^{-1} \text { curl curl } \boldsymbol{H}(x, t)=0 \\
\operatorname{div} \boldsymbol{H}(x, t)=0, \quad x \in \Omega_{1}, \quad t \in(0, T) \\
\operatorname{curl} \boldsymbol{H}(x, t)=0, \quad \operatorname{div} \boldsymbol{H}(x, t)=0, \quad x \in \Omega_{2}, \\
\boldsymbol{H}_{\tau}^{(1)}-\boldsymbol{H}_{\tau}^{(2)}=\boldsymbol{a}(x, t), \quad \mu_{1} \boldsymbol{H}^{(1)} \cdot \boldsymbol{n}-\mu_{2} \boldsymbol{H}^{(2)} \cdot \boldsymbol{n}=b(x, t), \quad x \in S, \\
\boldsymbol{H}^{(2)} \rightarrow 0, \quad|x| \rightarrow \infty \\
\boldsymbol{H}(x, 0)=0, \quad x \in \Omega_{1} \cup \Omega_{2} .
\end{array}\right.
$$

Here $\mu_{1}, \mu_{2}, \alpha$ are positive constants, $\boldsymbol{n}$ is the exterior unit normal to $S$ with respect to $\Omega_{1}, \boldsymbol{H}^{(i)}=\left.\boldsymbol{H}\right|_{x \in \bar{\Omega}_{i}}, \boldsymbol{H}_{\tau}^{(i)}=\boldsymbol{H}^{(i)}-\boldsymbol{n}\left(\boldsymbol{H}^{(i)} \cdot \boldsymbol{n}\right)$ is the tangential component of $\boldsymbol{H}^{(i)}$, $i=1,2, \boldsymbol{a}, b$ are given functions on $S \times(0, T)=S_{T}, \boldsymbol{a} \cdot \boldsymbol{n}=0$. For simplicity, we set $\alpha=\mu_{1}^{-1}$, so that the first equation in (1.1) becomes

$$
\boldsymbol{H}_{t}+\operatorname{curl} \operatorname{curl} \boldsymbol{H}=0 .
$$

We assume that $\boldsymbol{a}(x, 0)=0, b(x, 0)=0$.

Problems like (1.1) arise in the analysis of the problems of magnetohydrodynamics where the magnetic and electric fields should be found not only in the domain $\Omega_{1}$ filled with fluid but also in the surrounding vacuum region $\Omega_{2}$ (see [1, 2, 3]). In the present paper we obtain the classical solution of (1.1) by reducing this problem to a system of singular integral equations on $S$ of mixed type. In the case where $\Omega_{2}=\varnothing$, such a

Key words and phrases. Fredholm-Volterra singular integral equations, classical solution. 
reduction was made by Sh. Sahaev in [4], where a system of Volterra integral equations without singular integrals was obtained.

Since curl $\boldsymbol{H}=0$ and $\operatorname{div} \boldsymbol{H}=0$ in $\Omega_{2}, \boldsymbol{H}^{(2)}$ should be equal to the gradient of a harmonic function $\varphi$, and (1.1) can be viewed as a problem for $\boldsymbol{H}^{(1)} \equiv \boldsymbol{H}$ and $\varphi$ :

$$
\left\{\begin{array}{l}
\boldsymbol{H}_{t}(x, t)+\text { curl curl } \boldsymbol{H}(x, t)=0 \\
\operatorname{div} \boldsymbol{H}(x, t)=0, \quad x \in \Omega_{1}, \quad t \in(0, T) \\
\nabla^{2} \varphi(x, t)=0, \quad x \in \Omega_{2}, \\
\boldsymbol{n}(x) \times \boldsymbol{H}-\boldsymbol{n}(x) \times \nabla \varphi(x . t)=\boldsymbol{n}(x) \times \boldsymbol{a}(x, t), \\
\mu_{1} \boldsymbol{H} \cdot \boldsymbol{n}-\mu_{2} \frac{\partial \varphi}{\partial n}=b(x, t), \quad x \in S, \quad \varphi \rightarrow 0, \quad|x| \rightarrow \infty \\
\boldsymbol{H}(x, 0)=0, \quad x \in \Omega_{1} .
\end{array}\right.
$$

We seek $\boldsymbol{H}$ and $\varphi$ in the form of potentials

$$
\begin{aligned}
\boldsymbol{H}(x, t) & =\operatorname{curl} \int_{0}^{t} d \tau \int_{S} \Gamma(x-y, t-\tau) \cdot \boldsymbol{\lambda}(y, \tau) d S_{y} \\
& =\int_{0}^{t} d \tau \int_{S} \nabla \Gamma(x-y) \times \boldsymbol{\lambda}(y, \tau) d S_{y}, \quad x \in \bar{\Omega}_{1}, \\
\varphi(x, t) & =\int_{S} E(x-y) m(y, t) d S_{y}, \quad x \in \bar{\Omega}_{2},
\end{aligned}
$$

where $\Gamma$ and $E$ are fundamental solutions of the heat and Laplace equations, respectively:

$$
\Gamma(x, t)=\frac{1}{(4 \pi t)^{3 / 2}} e^{-\frac{|x|^{2}}{4 t}}, \quad E(x)=-\frac{1}{4 \pi|x|}, \quad x \in \mathbb{R}^{3}, \quad t>0 .
$$

Since

$$
\begin{aligned}
& \boldsymbol{n}(x) \times\left(\nabla_{x} \Gamma(x-y, t-\tau) \times \boldsymbol{\lambda}(y, \tau)\right) \\
& \quad=\nabla_{x} \Gamma(x-y, t-\tau)(\boldsymbol{n}(x) \cdot \boldsymbol{\lambda}(y, \tau))-\frac{\partial \Gamma(x-y, t-\tau)}{\partial n_{x}} \boldsymbol{\lambda}(y, \tau), \\
& \boldsymbol{n}(x) \cdot\left(\nabla_{x} \Gamma(x-y, t-\tau) \times \boldsymbol{\lambda}(y, \tau)\right)=\boldsymbol{\lambda}(y, \tau) \cdot\left(\boldsymbol{n}(x) \times \nabla_{x} \Gamma(x-y, t-\tau)\right),
\end{aligned}
$$

the boundary conditions imply

$$
\begin{aligned}
& \mathfrak{S} m+\frac{1}{2} \boldsymbol{\lambda}-\mathfrak{K} \boldsymbol{\lambda}=-\boldsymbol{n} \times \boldsymbol{a}, \\
& \mu_{1} \mathfrak{G} \boldsymbol{\lambda}-\mu_{2}\left(\mathfrak{V} m-\frac{1}{2} m\right)=b,
\end{aligned}
$$

where

$$
\begin{aligned}
\mathfrak{S} m & =\int_{S}(\boldsymbol{n}(x) \times \nabla E(x-y)) m(y, t) d S, \\
\mathfrak{V} m & =\int_{S} \frac{\partial E(x-y)}{\partial n} m(y, t) d S, \\
\mathfrak{G} \boldsymbol{\lambda} & =\int_{0}^{t} d \tau \int_{S}(\boldsymbol{n}(x) \times \nabla \Gamma(x-y, t-\tau)) \cdot \boldsymbol{\lambda}(y, \tau) d S, \\
\mathfrak{K} \boldsymbol{\lambda} & =\int_{0}^{t} d \tau \int_{S} \mathbf{K}(x, y, t-\tau) \cdot \boldsymbol{\lambda}(y, \tau) d S,
\end{aligned}
$$

with the matrix $\mathbf{K}$ given by

$$
\mathbf{K}(x, y, t-\tau)=\nabla_{x} \Gamma(x-y, t-\tau) \otimes(\boldsymbol{n}(x)-\boldsymbol{n}(y))-I \frac{\partial \Gamma(x-y, t-\tau)}{\partial n},
$$


provided

$$
\boldsymbol{\lambda}(y, t) \cdot \boldsymbol{n}(y)=0 .
$$

System (1.4) is a system of singular integral equations of mixed type on $S$. The singular operators $\mathfrak{S}$ and $\mathfrak{G}$ can be defined by

$$
\begin{aligned}
& \mathfrak{S} m(x, t)=\lim _{\epsilon \rightarrow 0} \int_{S^{\epsilon}} \boldsymbol{n}(x) \times \nabla E(x-y) m(y, t) d S, \\
& \mathfrak{G} \boldsymbol{\lambda}(x, t)=\lim _{\epsilon \rightarrow 0} \int_{0}^{t} d \tau \int_{S^{\epsilon}}(\boldsymbol{n}(x) \times \nabla \Gamma(x-y, t-\tau)) \cdot \boldsymbol{\lambda}(y, t) d S,
\end{aligned}
$$

where $S^{\epsilon}=\{y \in S:|x-y|>\epsilon\}$, and since the single layer harmonic and heat potentials are continuous on $S$, we have

$$
\lim _{z \rightarrow x \in S}(\mathfrak{S} m)(z, t)=(\mathfrak{S} m)(x, t), \quad \lim _{z \rightarrow x \in S}(\mathfrak{G} \boldsymbol{\lambda})(z, t)=(\mathfrak{G} \lambda)(x, t) .
$$

As was mentioned above, in the paper [4] a problem similar to (1.1) in the domain $\Omega_{1}$ (so that $\Omega_{2}=\varnothing$ ) was reduced to a system of Volterra integral equations on $S$ :

$$
2 \mathfrak{K} \boldsymbol{\lambda}-\boldsymbol{\lambda}=\boldsymbol{\omega}, \quad \boldsymbol{\omega} \cdot \boldsymbol{n}=0,
$$

and condition (1.7) is justified.

We seek a solution of (1.4) in the function space $C^{\alpha^{\prime}, 0}\left(S_{T}\right)$ with the norm

$$
\|u\|_{C^{\alpha^{\prime}, 0}\left(S_{T}\right)}=\sup _{t<T}\|u(\cdot, t)\|_{C^{\alpha^{\prime}(S)}},
$$

where $C^{\alpha^{\prime}}(S)$ is the space of Hölder continuous functions with the exponent $\alpha^{\prime} \in(0, \alpha)$ and $S_{T}=S \times(0, T)$. The main result of the paper is as follows.

Theorem 1. Let $S \in C^{1+\alpha}, \alpha^{\prime} \in(0, \alpha)$. For arbitrary $b \in C^{\alpha^{\prime}, 0}\left(S_{T}\right), \boldsymbol{a} \in C^{\alpha^{\prime}, 0}\left(S_{T}\right)$, such that $\boldsymbol{a} \cdot \boldsymbol{n}=0$, system (1.4) is uniquely solvable in $C^{\alpha^{\prime}, 0}\left(S_{T}\right)$, and

$$
\|\boldsymbol{\lambda}\|_{C^{\alpha^{\prime}, 0}\left(S_{T}\right)}+\|m\|_{C^{\alpha^{\prime}, 0}\left(S_{T}\right)} \leq c(T)\left(\|\boldsymbol{a}\|_{C^{\alpha^{\prime}, 0}\left(S_{T}\right)}+\|b\|_{C^{\alpha^{\prime}, 0}\left(S_{T}\right)}\right) .
$$

The vector $\boldsymbol{\lambda}$ satisfies

$$
\boldsymbol{\lambda}(x, t) \cdot \boldsymbol{n}(x)=0 .
$$

If $\boldsymbol{a}(x, 0)=0$ and $b(x, 0)=0$, then (1.3) is a classical solution of problem (1.2). It is unique, because in the case where $\boldsymbol{a}=0$ and $b=0$ the functions $\boldsymbol{H}$ and $\varphi$ vanish, see [2].

Methods for analyzing systems of singular integral equations on manifolds are provided by the theory of pseudo differential operators (see, e.g., the monograph [5]). To obtain our rather particular result, we use more elementary tools. Along with (1.4), we consider a simpler system, specifically,

$$
\mathfrak{S} m_{0}+\frac{1}{2} \boldsymbol{\lambda}_{0}=-\boldsymbol{n} \times \boldsymbol{a}, \quad \mu_{1} \mathfrak{G} \boldsymbol{\lambda}_{0}+\frac{\mu_{2}}{2} m_{0}=b,
$$

which is easily reduced to one equation

$$
\mathfrak{A} m_{0} \equiv-2 \mu_{1} \mathfrak{G S} m_{0}+\frac{\mu_{2}}{2} m_{0}=b+2 \mu_{1} \mathfrak{G}(\boldsymbol{n} \times \boldsymbol{a}) \equiv g .
$$

The following important auxiliary result concerns the solvability of system (1.11).

Theorem 2. Let $S \in C^{1+\alpha}, \alpha^{\prime} \in(0, \alpha)$. For arbitrary $b \in C^{\alpha^{\prime}, 0}\left(S_{T}\right)$ and $\boldsymbol{a} \in C^{\alpha^{\prime}, 0}\left(S_{T}\right)$ such that $\boldsymbol{a} \cdot \boldsymbol{n}=0$, system (1.11) is uniquely solvable in $C^{\alpha^{\prime}, 0}\left(S_{T}\right)$, and

$$
\left\|\boldsymbol{\lambda}_{0}\right\|_{C^{\alpha^{\prime}, 0}\left(S_{T}\right)}+\left\|m_{0}\right\|_{C^{\alpha^{\prime}, 0}\left(S_{T}\right)} \leq c(T)\left(\|\boldsymbol{a}\|_{C^{\alpha^{\prime}, 0}\left(S_{T}\right)}+\|b\|_{C^{\alpha^{\prime}, 0}\left(S_{T}\right)}\right) .
$$

The vector $\boldsymbol{\lambda}_{0}$ satisfies

$$
\boldsymbol{\lambda}_{0}(x, t) \cdot \boldsymbol{n}(x)=0 .
$$


In $\S 2$, we deduce Theorem 1 from Theorem 2, which is proved in $\S 3$. The proof is carried out with the construction of the regularizer of the operator $\mathcal{A}$, like it is done in the theory of elliptic and parabolic boundary-value problems. The regularizer is defined on the basis of solution of equation (1.12) in the case where $S=\mathbb{R}^{2}=\left\{x_{3}=0\right\}$. In this case, the operators $\mathfrak{G}, \mathfrak{S}$ are replaced with

$$
\begin{aligned}
\mathfrak{G}_{0} \boldsymbol{\lambda}_{0} & =\int_{0}^{t} d \tau \int_{\mathbb{R}^{2}}\left(\boldsymbol{e}_{3} \times \nabla \Gamma\left(x^{\prime}-y^{\prime}, 0, t-\tau\right)\right) \boldsymbol{\lambda}_{0}\left(y^{\prime}, \tau\right) d y^{\prime}, \\
\mathfrak{S}_{0} m_{0} & =\int_{\mathbb{R}^{2}}\left(\boldsymbol{e}_{3} \times \nabla E\left(x^{\prime}-y^{\prime}, 0\right)\right) m_{0}\left(y^{\prime}, \tau\right) d y^{\prime}, \quad x^{\prime}=\left(x_{1}, x_{2}\right) \in \mathbb{R}^{2} .
\end{aligned}
$$

The rest of this section is devoted to the proof of the solvability of equation (1.12) on $\mathbb{R}^{2}$ and of the continuity of the operator $\mathfrak{A}$ in $C^{\alpha^{\prime}, 0}\left(S_{T}\right)$. Making the Fourier-Laplace transformation with respect to $x^{\prime}, t$, defined by

$$
F u \equiv \widetilde{u}(\xi, s)=\int_{0}^{\infty} d t \int_{\mathbb{R}^{2}} e^{-s t-i \xi \cdot x^{\prime}} u\left(x^{\prime}, t\right) d x^{\prime}, \quad \operatorname{Re} s \geq 0,
$$

we convert equation $(1.12)$ on $\mathbb{R}^{2}$ into

$$
\left(\frac{\mu_{1}}{2} \frac{|\xi|}{r}+\frac{\mu_{2}}{2}\right) \tilde{m}_{0}(\xi, s)=\tilde{g}(\xi, s), \quad r=\sqrt{s+|\xi|^{2}},
$$

or

$$
\frac{\mu_{2}}{2} m_{0}\left(x^{\prime}, t\right)+\int_{0}^{t} d \tau \int_{\mathbb{R}^{2}} K\left(x^{\prime}-y^{\prime}, t-\tau\right) m_{0}\left(y^{\prime}, \tau\right) d y^{\prime}=g\left(x^{\prime}, t\right),
$$

with the kernel $K$ defined by $\widetilde{K}(\xi, s)=\frac{\mu_{1}}{2} \frac{|\xi|}{r}$. Hence,

$$
\tilde{m}_{0}=\frac{2 r \tilde{g}}{\mu_{1}|\xi|+\mu_{2} r}=\frac{2 \widetilde{g}}{\mu_{2}}-\frac{2 \widetilde{g} \mu_{1}}{\mu_{2}} \frac{|\xi|}{\mu_{1}|\xi|+\mu_{2} r},
$$

i.e.,

$$
m_{0}\left(x^{\prime}, t\right)=\frac{2}{\mu_{2}} g\left(x^{\prime}, t\right)-\frac{2 \mu_{1}}{\mu_{2}} \int_{0}^{t} d \tau \int_{\mathbb{R}^{2}} L\left(x^{\prime}-y^{\prime}, t-\tau\right) g\left(y^{\prime}, \tau\right) d y^{\prime},
$$

where $\widetilde{L}=\frac{|\xi|}{\mu_{1}|\xi|+\mu_{2} r}$. As was shown in [6, Proposition 2.1],

$$
\begin{gathered}
\left|D_{x^{\prime}}^{j} L\left(x^{\prime}, t\right)\right| \leq c \frac{1}{\sqrt{t}\left(\left|x^{\prime}\right|^{2}+t\right)^{3 / 2+|j| / 2}}, \\
\left|D_{x^{\prime}}^{j} K\left(x^{\prime}, t\right)\right| \leq c \frac{1}{\sqrt{t}\left(\left|x^{\prime}\right|^{2}+t\right)^{3 / 2+|j| / 2}} ;
\end{gathered}
$$

and, moreover,

$$
\int_{\mathbb{R}^{2}} L\left(x^{\prime}, t\right) d x^{\prime}=\int_{\mathbb{R}^{2}} K\left(x^{\prime}, t\right) d x^{\prime}=0 .
$$

Proposition 1. The function

$$
u\left(x^{\prime}, t\right)=\int_{0}^{t} d \tau \int_{\mathbb{R}^{2}} L\left(x^{\prime}-y^{\prime}, t-\tau\right) g\left(y^{\prime}, \tau\right) d y^{\prime}
$$

satisfies the inequalities

$$
\begin{aligned}
& \|u\|_{\dot{C}^{\alpha^{\prime}, 0}\left(\mathbb{R}_{T}\right)} \leq c\|g\|_{\dot{C}^{\alpha^{\prime}}\left(\mathbb{R}_{T}\right)}, \\
& \sup _{\mathbb{R}_{t}}\left|u\left(x^{\prime}, t\right)\right| \leq c \int_{0}^{t}\|g\|_{\dot{C}^{\alpha^{\prime}}\left(\mathbb{R}^{2}\right)} \frac{d \tau}{(t-\tau)^{1-\alpha^{\prime} / 2}}
\end{aligned}
$$


with $c$ independent of $T$, where $0<t \leq T, \alpha^{\prime} \in(0,1), \mathbb{R}_{T}=\mathbb{R}^{2} \times(0, T)$, and

$$
\|u\|_{\dot{C}^{\alpha^{\prime}, 0}\left(\mathbb{R}_{T}\right)}=\sup _{x^{\prime}, y^{\prime} \in \mathbb{R}^{2}} \sup _{t<T} \frac{\left|u\left(x^{\prime}, t\right)-u\left(y^{\prime}, t\right)\right|}{\left|x^{\prime}-y^{\prime}\right|^{\alpha^{\prime}}} .
$$

The function

$$
v\left(x^{\prime} t\right)=\int_{0}^{t} d \tau \int_{\mathbb{R}^{2}} K\left(x^{\prime}-y^{\prime}, t-\tau\right) m_{0}\left(y^{\prime}, \tau\right) d y^{\prime}
$$

satisfies the same estimates.

The proof of the first inequality in (1.20) was given in [6, Proposition 2.2], and the second estimate is obtained as follows: by (1.19), we have

$$
u\left(x^{\prime}, t\right)=\int_{0}^{t} d \tau \int_{\mathbb{R}^{2}} L\left(x^{\prime}-y^{\prime}, t-\tau\right)\left(g\left(y^{\prime}, \tau\right)-g\left(x^{\prime}, \tau\right)\right) d y^{\prime},
$$

and, by (1.18),

$$
\begin{aligned}
\left|u\left(x^{\prime}, t\right)\right| & \leq c \int_{0}^{t} \frac{\|g\|_{\dot{C}^{\alpha^{\prime}}\left(\mathbb{R}^{2}\right)}}{\sqrt{t-\tau}} d \tau \int_{\mathbb{R}^{2}} \frac{\left|x^{\prime}-y^{\prime}\right|^{\alpha^{\prime}} d y^{\prime}}{\left(\left|x^{\prime}-y^{\prime}\right|^{2}+t-\tau\right)^{3 / 2}} \\
& \leq c \int_{0}^{t}\|g\|_{\dot{C}^{\alpha^{\prime}}\left(\mathbb{R}^{2}\right)} \frac{d \tau}{(t-\tau)^{1-\alpha^{\prime} / 2}} .
\end{aligned}
$$

Hence, the operator $\mathfrak{A}_{0}=-2 \mu_{1} \mathfrak{G}_{0} \mathfrak{S}_{0}+\frac{\mu_{2}}{2} I$ maps $C^{\alpha^{\prime}, 0}\left(\mathbb{R}_{T}\right)$ onto itself, is continuous, and has a continuous inverse.

In conclusion, we show that $\mathfrak{A}=-2 \mu_{1} \mathfrak{G} \mathfrak{S}+\frac{\mu_{2}}{2} I$ is a continuous operator in $C^{\alpha^{\prime}, 0}\left(S_{T}\right)$.

Proposition 2. If $f \in C^{\alpha^{\prime}}(S), \alpha^{\prime} \in(0, \alpha]$, then

$$
\|\mathfrak{S} f\|_{C^{\alpha^{\prime}(S)}} \leq c\|f\|_{C^{\alpha^{\prime}(S)}} .
$$

If $\boldsymbol{h} \in C^{\alpha^{\prime}, 0}\left(S_{T}\right)$, then

$$
\|\mathfrak{G} h\|_{C^{\alpha^{\prime}, 0}\left(S_{T}\right)} \leq c\|\boldsymbol{h}\|_{C^{\alpha^{\prime}, 0}\left(S_{T}\right)} .
$$

Proof. Inequality (1.21) is known from the classical theory of potentials [7]. The estimate of $\sup _{S_{T}}|\mathfrak{G} \boldsymbol{h}|$ is easily deduced from the formula

$$
\begin{aligned}
\mathfrak{G} \boldsymbol{h}(x, t) & =\int_{0}^{t} d \tau \int_{S}((\boldsymbol{n}(x)-\boldsymbol{n}(y)) \times \nabla \Gamma(x-y, t-\tau)) \cdot \boldsymbol{h}(y, \tau) d S \\
& +\int_{0}^{t} d \tau \int_{S}(\boldsymbol{n}(y) \times \nabla \Gamma(x-y, t-\tau)) \cdot(\boldsymbol{h}(y, \tau)-\boldsymbol{h}(x, \tau)) d S
\end{aligned}
$$

(we have taken into account that $\int_{S} \boldsymbol{n}(y) \times \nabla \Gamma(x-y, t-\tau) d S=0$ ):

$$
\begin{aligned}
|\mathfrak{G} \boldsymbol{h}(x, t)| & \leq c \int_{0}^{t}\|\boldsymbol{h}(\cdot, \tau)\|_{C^{\alpha^{\prime}(S)}} d \tau \int_{S} \frac{d S}{\left(|x-y|^{2}+(t-\tau)\right)^{2-\alpha^{\prime} / 2}} \\
& \leq c \int_{0}^{t}\|\boldsymbol{h}(\cdot, \tau)\|_{C^{\alpha^{\prime}}(S)} \frac{d \tau}{(t-\tau)^{1-\alpha^{\prime} / 2}} \\
& \leq c t^{\alpha^{\prime} / 2} \sup _{\tau<t}\|\boldsymbol{h}(\cdot, \tau)\|_{C^{\alpha^{\prime}}(S)} .
\end{aligned}
$$

Next, we estimate $|u(x, t)-u(z, t)|, u=\mathfrak{G} \boldsymbol{h}, x, z \in S$. It suffices to assume that $x$ and $z$ are close to each other: $|x-z| \equiv r \leq d / 2$, where $d$ is the radius of the Lyapunov 
sphere. Let $\sigma_{r}=\{y \in S:|x-y| \leq 2 r\}, \boldsymbol{F}(x, y)=\boldsymbol{n}(x) \times \nabla \Gamma(x-y, t)$. We have

$$
\begin{aligned}
u(x, t) & -u(z, t)=\int_{0}^{t} d \tau \int_{\sigma_{r}} \boldsymbol{F}(x, y, t-\tau) \cdot(\boldsymbol{h}(y, \tau)-\boldsymbol{h}(x, \tau)) d S \\
& -\int_{0}^{t} d \tau \int_{\sigma_{r}} \boldsymbol{F}(z, y, t-\tau) \cdot(\boldsymbol{h}(y, \tau)-\boldsymbol{h}(z, \tau)) d S \\
& +\int_{0}^{t}(\boldsymbol{h}(x, \tau)-\boldsymbol{h}(z, \tau)) d \tau \cdot \int_{\sigma_{r}} \boldsymbol{F}(x, y, t-\tau) d S \\
& +\int_{0}^{t} d \tau \int_{S \backslash \sigma_{r}}(\boldsymbol{F}(x, y, t-\tau)-\boldsymbol{F}(z, y, t-\tau)) \cdot(\boldsymbol{h}(y, \tau)-\boldsymbol{h}(z, \tau)) d S \\
& +\int_{0}^{t} \boldsymbol{h}(z, \tau) d \tau \cdot \int_{S}(\boldsymbol{F}(x, y, t-\tau)-\boldsymbol{F}(z, y, t-\tau)) d S \equiv \sum_{i=1}^{5} U_{i}, \\
\left|U_{1}\right| \leq & \|\boldsymbol{h}\|_{\dot{C}^{\alpha^{\prime}, 0}\left(S_{t}\right)} \int_{0}^{t} d \tau \int_{\sigma_{r}}|x-y|^{\alpha^{\prime}}|\boldsymbol{F}(x, y, t-\tau)| d S \leq c r^{\alpha^{\prime}}\|\boldsymbol{h}\|_{\dot{C}^{\alpha^{\prime}, 0}\left(S_{t}\right)} .
\end{aligned}
$$

In the same way we obtain

$$
\left|U_{2}\right| \leq c r^{\alpha^{\prime}}\|\boldsymbol{h}\|_{\dot{C}^{\alpha^{\prime}, 0}\left(S_{t}\right)}
$$

To estimate $U_{3}$, we consider

$$
\begin{aligned}
\int_{\sigma_{r}} \boldsymbol{F}(x, y, t-\tau) d S=\int_{\sigma_{r}} & (\boldsymbol{n}(x)-\boldsymbol{n}(y)) \times \nabla \Gamma(x-y, t-\tau) d S \\
& +\int_{S \backslash \sigma_{r}} \boldsymbol{n}(y) \times \nabla \Gamma(x-y, t-\tau) d S=I_{1}+I_{2} .
\end{aligned}
$$

Observe that

$$
\left|I_{1}\right| \leq c \int_{\sigma_{r}} \frac{d S}{\left(|x-y|^{2}+t-\tau\right)^{2-\alpha / 2}}
$$

and, in view of the Stokes formula,

$$
\int_{S \backslash \sigma_{r}}\left(n_{i}(y) \frac{\partial \Gamma(x-y, t-\tau)}{\partial y_{j}}-n_{j}(y) \frac{\partial \Gamma(x-y, t-\tau)}{\partial y_{i}}\right) d S=\int_{\partial \sigma_{r}} \Gamma(x-y, t-\tau) d y_{k},
$$

where $(i, j, k)$ is a cyclic permutation of $(1,2,3)$ and the last integral is taken over the contour $\partial \sigma_{r}$ that bounds $\sigma_{r}$. Hence,

$$
\left|I_{2}\right| \leq c \int_{\partial \sigma_{r}} \frac{d l}{\left(r^{2}+t-\tau\right)^{3 / 2}}
$$

and

$$
\left|U_{3}\right| \leq c r^{\alpha^{\prime}}\|\boldsymbol{h}\|_{\dot{C}^{\alpha^{\prime}, 0}\left(S_{t}\right)} \int_{0}^{t}\left(\left|I_{1}\right|+\left|I_{2}\right|\right) d \tau \leq c r^{\alpha^{\prime}}\|\boldsymbol{h}\|_{\dot{C}^{\alpha^{\prime}, 0}\left(S_{t}\right)} .
$$

We also have

$$
\begin{aligned}
\left|U_{4}\right| \leq c\|\boldsymbol{h}\|_{\dot{C}^{\alpha^{\prime}, 0}\left(S_{t}\right)}\left(r^{\alpha^{\prime}} \int_{0}^{t} d \tau \int_{S \backslash \sigma_{r}} \frac{d S}{\left(|x-y|^{2}+t-\tau\right)^{2-\alpha^{\prime} / 2}}\right. \\
\left.+r \int_{0}^{t} d \tau \int_{S \backslash \sigma_{r}} \frac{d S}{\left(|x-y|^{2}+t-\tau\right)^{5 / 2-\alpha^{\prime} / 2}}\right) \leq c r^{\alpha^{\prime}}\|\boldsymbol{h}\|_{\dot{C}^{\alpha^{\prime}, 0}\left(S_{t}\right)},
\end{aligned}
$$




$$
\begin{aligned}
& U_{5}=\int_{0}^{t} \boldsymbol{h}(z, \tau) d \tau \cdot \int_{S}((\boldsymbol{n}(x)-\boldsymbol{n}(y)) \times \nabla \Gamma(x-y, t-\tau)-(\boldsymbol{n}(z)-\boldsymbol{n}(y)) \times \nabla \Gamma(z-y, t-\tau)) d S \\
&=\int_{0}^{t} \boldsymbol{h}(z, \tau) d \tau \cdot \int_{\sigma_{r}}((\boldsymbol{n}(x)-\boldsymbol{n}(y)) \times \nabla \Gamma(x-y, t-\tau) \\
&\quad-(\boldsymbol{n}(z)-\boldsymbol{n}(y)) \times \nabla \Gamma(z-y, t-\tau)) d S \\
&+\int_{0}^{t} \boldsymbol{h}(z, \tau) d \tau \int_{S \backslash \sigma_{r}}((\boldsymbol{n}(z)-\boldsymbol{n}(y)) \times(\nabla \Gamma(x-y, t-\tau)-\nabla \Gamma(z-y, t-\tau)) d S \\
&+\int_{0}^{t} \boldsymbol{h}(z, \tau) d \tau \cdot(\boldsymbol{n}(x)-\boldsymbol{n}(z)) \times \int_{S \backslash \sigma_{r}} \nabla \Gamma(x-y, t-\tau) d S=W_{1}+W_{2}+W_{3} .
\end{aligned}
$$

The first two integrals $W_{1}$ and $W_{2}$ on the right hand side are estimated by

$$
c r^{\alpha^{\prime}} \sup _{S_{t}}|\boldsymbol{h}(y, \tau)|
$$

Moreover, since

$$
\begin{aligned}
& \int_{S \backslash \sigma_{r}} \nabla_{y} \Gamma(x-y, t-\tau) d S \\
&=\int_{S \backslash \sigma_{r}} \boldsymbol{n}(y) \frac{\partial \Gamma(x-y, t-\tau)}{\partial n_{y}} d S \\
& \quad-\int_{S \backslash \sigma_{r}}((\boldsymbol{n}(y)-\boldsymbol{n}(x)) \times(\boldsymbol{n}(y) \times \nabla \Gamma(x-y, t-\tau)) \\
&+\boldsymbol{n}(x) \times(\boldsymbol{n}(y) \times \nabla \Gamma(x-y, t-\tau))) d S
\end{aligned}
$$

and

$$
\int_{0}^{t} d \tau\left|\int_{S \backslash \sigma_{r}} \boldsymbol{n}(y) \times \nabla \Gamma(x-y, t-\tau) d S\right| \leq c \int_{0}^{t} \int_{\partial \sigma_{r}} \frac{d l}{\left(r^{2}+t-\tau\right)^{3 / 2}} \leq c
$$

we conclude that the integral $W_{3}$ is also controlled by $c r^{\alpha^{\prime}} \sup _{S_{t}}|\boldsymbol{h}(y, \tau)|$. This completes the proof of Proposition 2.

By (1.21) and (1.22),

$$
\|\mathfrak{A} \boldsymbol{h}\|_{C^{\alpha^{\prime}, 0}\left(S_{T}\right)} \leq c\|\boldsymbol{h}\|_{C^{\alpha^{\prime}, 0}\left(S_{T}\right)} .
$$

We complete Proposition 2 with the following result.

Proposition 3. If $\boldsymbol{h} \in C^{\alpha^{\prime \prime}, 0}\left(S_{T}\right)$ and $0<\alpha^{\prime}<\alpha^{\prime \prime} \leq \alpha$, then

$$
\begin{aligned}
\|\mathfrak{G} h\|_{C^{\alpha^{\prime}, 0}\left(S_{t}\right)} & \leq c \int_{0}^{t}\|\boldsymbol{h}\|_{C^{\alpha^{\prime \prime}}(S)} \frac{d \tau}{(t-\tau)^{1-\epsilon^{\prime \prime} / 2}}, \quad \epsilon^{\prime \prime}=\alpha^{\prime \prime}-\alpha^{\prime} \\
\|\mathfrak{K} \boldsymbol{\lambda}\|_{C^{\alpha^{\prime}, 0}\left(S_{t}\right)} & \leq c \int_{0}^{t} \sup _{S_{\tau}}|\boldsymbol{\lambda}(y, \tau)| \frac{d \tau}{(t-\tau)^{1-\epsilon^{\prime \prime} / 2}} .
\end{aligned}
$$


Proof. The estimate for $\sup _{S}|\mathfrak{G} \boldsymbol{h}|$ follows from (1.24). The difference $(\mathfrak{G} \boldsymbol{h})(x, t)-$ $(\mathfrak{G} \boldsymbol{h})(y, t)$ is estimated by using (1.25). Instead of (1.26)-(1.31), we have

$$
\begin{aligned}
\left|U_{1}\right| & \leq c \int_{0}^{t}\|\boldsymbol{h}\|_{C^{\alpha^{\prime \prime}(S)}} d \tau \int_{\sigma_{r}}|x-y|^{\alpha^{\prime \prime}}|\boldsymbol{F}| d S \leq c r^{\alpha^{\prime}} \int_{0}^{t}\|\boldsymbol{h}\|_{C^{\alpha^{\prime \prime}}(S)} \frac{d \tau}{(t-\tau)^{1-\epsilon^{\prime \prime} / 2}} \\
\left|U_{2}\right| & \leq c r^{\alpha^{\prime}} \int_{0}^{t}\|\boldsymbol{h}\|_{C^{\alpha^{\prime \prime}(S)}} \frac{d \tau}{(t-\tau)^{1-\epsilon^{\prime \prime} / 2}}, \\
\left|U_{3}\right| & \leq c r^{\alpha^{\prime}} \int_{0}^{t}\|\boldsymbol{h}\|_{C^{\alpha^{\prime \prime}(S)}}\left(\left|I_{1}\right|+\left|I_{2}\right|\right) d \tau \leq c r^{\alpha^{\prime}} \int_{0}^{t}\|\boldsymbol{h}\|_{C^{\alpha^{\prime \prime}(S)} \frac{d \tau}{(t-\tau)^{1-\epsilon^{\prime \prime} / 2}}} \\
\left|U_{4}\right| & \leq \int_{0}^{t}\|\boldsymbol{h}\|_{\dot{C}^{\alpha^{\prime \prime}(S)}}\left(r^{\alpha} \int_{0}^{t} d \tau \int_{S \backslash \sigma_{r}} \frac{d S}{\left(|x-y|^{2}+t-\tau\right)^{2-\alpha^{\prime \prime} / 2}}\right. \\
& \left.\leq c \int_{0}^{\alpha^{\prime}} \int_{0}^{t}\|\boldsymbol{h}\|_{C^{\alpha^{\prime \prime}}\left(S_{\tau}\right)} \frac{d \tau \int_{S \backslash \sigma_{r}} \frac{d \tau}{(t-\tau)^{1-\epsilon^{\prime \prime} / 2}},}{\left(|x-y|^{2}+t-\tau\right)^{5 / 2-\alpha^{\prime \prime} / 2}}\right) \\
\left|U_{5}\right| & \leq c r^{\alpha^{\prime}} \int_{0}^{t} \sup _{S}|\boldsymbol{h}(\cdot, \tau)| \frac{d \tau}{(t-\tau)^{1-\epsilon^{\prime \prime} / 2}} .
\end{aligned}
$$

These inequalities prove (1.32).

Estimate (1.33) is proved by the same arguments, but since $\mathbf{K}$ is weakly singular, we should employ the formula

$$
\begin{aligned}
(\mathfrak{K} \boldsymbol{\lambda})(x, t)-(\mathfrak{K} \boldsymbol{\lambda})(z, t) & \\
=\int_{0}^{t} d \tau \int_{\sigma_{r}} \mathbf{K}(x, y, t & -\tau) \boldsymbol{\lambda}(y, \tau) d S-\int_{0}^{t} d \tau \int_{\sigma_{r}} \mathbf{K}(z, y, t-\tau) \boldsymbol{\lambda}(y, \tau) d S \\
& +\int_{0}^{t} d \tau \int_{S \backslash \sigma_{r}}(\mathbf{K}(x, y, t-\tau)-\mathbf{K}(z, y, t-\tau)) \boldsymbol{\lambda}(y, \tau) d S
\end{aligned}
$$

instead of $(1.25)$.

\section{$\S 2$. Proof of Theorem 1}

In this section, we deduce Theorem 1 from Theorem 2. Suppose we have solved equations (1.11) and obtained estimate (1.13). Subtracting (1.11) from (1.4), we obtain

$$
\begin{aligned}
\mathfrak{S}\left(m-m_{0}\right)+\frac{1}{2}\left(\lambda-\lambda_{0}\right) & =\mathfrak{K}\left(\lambda-\lambda_{0}\right)+\mathfrak{K} \lambda_{0}, \\
\mu_{1} \mathfrak{G}\left(\boldsymbol{\lambda}-\boldsymbol{\lambda}_{0}\right)+\frac{\mu_{2}}{2}\left(m-m_{0}\right) & =\mu_{2} \mathfrak{V}\left(m-m_{0}\right)+\mu_{2} \mathfrak{V} m_{0} .
\end{aligned}
$$

Since $\mathfrak{K}$ is the Volterra operator with a weak singularity, the inverse operator $(I-$ $2 \mathfrak{K})^{-1}=I+\mathcal{R}$ exists, where the resolvent $\mathcal{R}$ is also weakly singular, whence

$$
\boldsymbol{\lambda}-\boldsymbol{\lambda}_{0}=(I+\mathcal{R})\left(-2 \mathfrak{S}\left(m-m_{0}\right)+2 \mathfrak{K} \boldsymbol{\lambda}_{0}\right),
$$

and the second equation in (2.1) takes the form

$$
\begin{aligned}
- & 2 \mu_{1} \mathfrak{G} \mathfrak{S}\left(m-m_{0}\right)+\frac{\mu_{2}}{2}\left(m-m_{0}\right) \\
& =\mu_{2} \mathfrak{V}\left(m-m_{0}\right)+\mu_{2} \mathfrak{V} m_{0}+2 \mu_{1} \mathfrak{G} \mathcal{R} \mathfrak{S}\left(m-m_{0}\right)-2 \mu_{1} \mathfrak{G}(I+\mathcal{R}) \mathfrak{K} \boldsymbol{\lambda}_{0} .
\end{aligned}
$$

For technical reasons, it is convenient to introduce a new unknown function

$$
q=\left(m-m_{0}\right)-2 \mathfrak{V}\left(m-m_{0}\right) .
$$


This equation is uniquely solvable with respect to $m-m_{0}$ for arbitrary $q \in C^{\alpha^{\prime}}(S)$ with $\alpha^{\prime} \in(0, \alpha]$, or even $q \in C(S)$, and

$$
\begin{aligned}
& c_{1}\|q\|_{C^{\alpha^{\prime}}(S)} \leq\left\|m-m_{0}\right\|_{C^{\alpha^{\prime}}(S)} \leq c_{2}\|q\|_{C^{\alpha^{\prime}}(S)}, \\
& c_{3} \sup _{S}|q(x)| \leq \sup _{S}\left|m-m_{0}\right| \leq c_{4} \sup _{S}|q(x)|
\end{aligned}
$$

(see [7]). We have

$$
m-m_{0}=\left(I+\mathcal{R}_{0}\right) q,
$$

where $\mathcal{R}_{0}$ is a weakly singular Fredholm operator (like $\mathfrak{V}$ ). Hence, (2.3) reduces to (2.6) $-2 \mu_{1} \mathfrak{G S S} q+\frac{\mu_{2}}{2} q=2 \mu_{1} \mathfrak{G}\left(\mathcal{R} \mathfrak{S}\left(m-m_{0}\right)+\mathfrak{S} \mathcal{R}_{0} q\right)+\mu_{2} \mathfrak{V} m_{0}-2 \mu_{1} \mathfrak{G}(I+\mathcal{R}) \mathfrak{K} \boldsymbol{\lambda}_{0}$, where $m-m_{0}$ is related to $q$ by (2.4). The operators $\mathcal{R}$ and $\mathcal{R}_{0}$ are smoothing, i.e.,

$$
\left\|\mathcal{R}_{0} f\right\|_{C^{\alpha^{\prime}}(S)} \leq c \sup _{S}|f(y)|, \quad\|\mathcal{R} \boldsymbol{h}\|_{C^{\alpha^{\prime}, 0}\left(S_{t}\right)} \leq c \int_{0}^{t} \sup _{S_{\tau}}|\boldsymbol{h}(y, \tau)| \frac{d \tau}{(t-\tau)^{1-\epsilon^{\prime} / 2}}
$$

where $\alpha^{\prime}<\alpha, \epsilon^{\prime}=\alpha-\alpha^{\prime}$. This estimate of $\mathcal{R}_{0} f$ is well known from the theory of potentials, and the second inequality in (2.7) follows from the representation of $\mathcal{R}$ in the form of the series

$$
\mathcal{R} \boldsymbol{h}=2 \mathfrak{K} \boldsymbol{h}+4 \mathfrak{K}^{2} \boldsymbol{h}+\ldots,
$$

similar to (2.11). Hence,

$$
\begin{aligned}
\left\|\mathfrak{G R S}\left(m-m_{0}\right)\right\|_{C^{\alpha^{\prime}, 0}\left(S_{t}\right)} & \leq c \int_{0}^{t}\left\|\mathcal{R} \mathfrak{S}\left(m-m_{0}\right)\right\|_{C^{\alpha^{\prime \prime}}(S)} \frac{d \tau}{(t-\tau)^{1-\epsilon^{\prime \prime} / 2}} \\
& \leq c \int_{0}^{t} \sup _{S_{\tau}}\left|\mathfrak{S}\left(m-m_{0}\right)\right| \frac{d \tau}{(t-\tau)^{1-\epsilon^{\prime \prime} / 2}} \\
& \leq c \int_{0}^{t}\|q\|_{C^{\alpha^{\prime}, 0}\left(S_{\tau}\right)} \frac{d \tau}{(t-\tau)^{1-\epsilon^{\prime \prime} / 2}}
\end{aligned}
$$

where $\alpha^{\prime}<\alpha^{\prime \prime}<\alpha$ and

$$
\begin{aligned}
\left\|\mathfrak{G S S} \mathcal{R}_{0} q\right\|_{C^{\alpha^{\prime}, 0}\left(S_{t}\right)} & \leq c \int_{0}^{t}\left\|\mathfrak{S} \mathcal{R}_{0} q\right\|_{C^{\alpha^{\prime \prime}}(S)} \frac{d \tau}{(t-\tau)^{1-\epsilon^{\prime \prime} / 2}} \\
& \leq c \int_{0}^{t}\left\|\mathcal{R}_{0} q\right\|_{C^{\alpha^{\prime \prime}(S)}} \frac{d \tau}{(t-\tau)^{1-\epsilon^{\prime \prime} / 2}} \\
& \leq c \int_{0}^{t} \sup _{S}|q| \frac{d \tau}{(t-\tau)^{1-\epsilon^{\prime \prime} / 2}} .
\end{aligned}
$$

Now, we pass to the proof of the solvability of equation (2.6) in the space $C^{\alpha^{\prime}, 0}\left(S_{T}\right)$. By Theorem 2, we can write (2.6) as

$$
q=\mathfrak{D} q+q_{0}
$$

where

$$
\begin{aligned}
\mathfrak{D} q & =\left(I-\frac{4 \mu_{1}}{\mu_{2}} \mathfrak{G} \mathfrak{S}\right)^{-1} \frac{4 \mu_{1}}{\mu_{2}} \mathfrak{G}\left(\mathcal{R S}\left(m-m_{0}\right)+\mathfrak{S} \mathcal{R}_{0} q\right), \\
q_{0} & =\left(I-\frac{4 \mu_{1}}{\mu_{2}} \mathfrak{G} \mathfrak{S}\right)^{-1}\left(2 \mathfrak{V} m_{0}-\frac{4 \mu_{1}}{\mu_{2}} \mathfrak{G}(I+\mathcal{R}) \mathfrak{K} \boldsymbol{\lambda}_{0}\right) .
\end{aligned}
$$

Proposition 2 and inequalities (2.8), (2.9), (1.13) show that

$$
\begin{aligned}
\left\|q_{0}\right\|_{C^{\alpha^{\prime}, 0}\left(S_{t}\right)} & \leq c\left(\left\|m_{0}\right\|_{C^{\alpha^{\prime}, 0}\left(S_{t}\right)}+\left\|\lambda_{0}\right\|_{C^{\alpha^{\prime}, 0}\left(S_{t}\right)}\right) \leq c\left(\|\boldsymbol{a}\|_{C^{\alpha^{\prime}, 0}\left(S_{t}\right)}+\|b\|_{C^{\alpha^{\prime}, 0}\left(S_{T}\right)}\right), \\
\|\mathfrak{D} q\|_{C^{\alpha^{\prime}, 0}\left(S_{t}\right)} & \leq c \int_{0}^{t}\|q\|_{C^{\alpha^{\prime}, 0}\left(S_{\tau}\right)} \frac{d \tau}{(t-\tau)^{1-\epsilon / 2}} .
\end{aligned}
$$


As in the classical theory of the Volterra integral equations (see [7]), we seek a solution of (2.10) in the form of the series

$$
q=q_{0}+\mathfrak{D} q_{0}+\mathfrak{D}^{2} q_{0}+\ldots
$$

It suffices to prove the convergence of this series in the norm of $C^{\alpha^{\prime}, 0}\left(S_{t}\right)$. We have

$$
\begin{aligned}
\left\|\mathfrak{D}^{2} q_{0}\right\|_{C^{\alpha^{\prime}, 0}\left(S_{t}\right)} & \leq C \int_{0}^{t}\left\|\mathfrak{D} q_{0}\right\|_{C^{\alpha^{\prime}, 0}\left(S_{\tau}\right)} \frac{d \tau}{(t-\tau)^{1-\epsilon / 2}} \\
& \leq C^{2} \int_{0}^{t} \frac{d \tau_{1}}{\left(t-\tau_{1}\right)^{1-\epsilon / 2}} \int_{0}^{\tau_{1}}\left\|q_{0}\right\|_{C^{\alpha^{\prime}, 0}\left(S_{\tau_{2}}\right)} \frac{d \tau_{2}}{\left(\tau_{1}-\tau_{2}\right)^{1-\epsilon / 2}} \\
& \leq C^{2}\left\|q_{0}\right\|_{C^{\alpha^{\prime}, 0}\left(S_{t}\right)} B(1, \epsilon / 2) \int_{0}^{t} \frac{\tau_{1}^{\epsilon / 2} d \tau_{1}}{\left(t-\tau_{1}\right)^{1-\epsilon / 2}} \\
& =C^{2} B(1, \epsilon / 2) B(1+\epsilon / 2, \epsilon / 2) t^{2 \epsilon / 2}\left\|q_{0}\right\|_{C^{\alpha^{\prime}, 0}\left(S_{t}\right)} \\
& =C^{2} t^{2 \epsilon / 2} \frac{\Gamma^{2}(\epsilon / 2)}{\Gamma(1+2 \epsilon / 2)}\left\|q_{0}\right\|_{C^{\alpha^{\prime}, 0}\left(S_{t}\right)}
\end{aligned}
$$

and, in general,

$$
\left\|\mathfrak{D}^{j} q_{0}\right\|_{C^{\alpha^{\prime}, 0}\left(S_{t}\right)} \leq C^{j} t^{j \epsilon / 2} \frac{\Gamma^{j}(\epsilon / 2)}{\Gamma(1+j \epsilon / 2)}\left\|q_{0}\right\|_{C^{\alpha^{\prime}, 0}\left(S_{t}\right)},
$$

where $B(p, q)=\frac{\Gamma(p) \Gamma(q)}{\Gamma(p+q)}$ and $\Gamma(p)$ is the Euler gamma-function (see [8]).

Since the series $\sum_{j=1}^{\infty} C^{j} t^{j \epsilon / 2} \frac{\Gamma^{j}(\epsilon / 2)}{\Gamma(1+j \epsilon / 2)}$ is convergent, so is the series $(2.11)$, and we obtain

$$
\|q\|_{C^{\alpha^{\prime}, 0}\left(S_{T}\right)} \leq c(T)\left\|q_{0}\right\|_{C^{\alpha^{\prime}, 0}\left(S_{T}\right)} .
$$

It is easily verified that the solvability of (2.10) implies the solvability of (2.6) and (1.11); moreover, we have

$$
\left\|q_{0}\right\|_{C^{\alpha^{\prime}, 0}\left(S_{T}\right)} \leq c\left(\|\boldsymbol{a}\|_{C^{\alpha^{\prime}, 0}\left(S_{T}\right)}+\|b\|_{C^{\alpha^{\prime}, 0}\left(S_{T}\right)}\right) .
$$

Theorem 1 is proved, now we need to prove Theorem 2 .

\section{§3. Proof of Theorem 2}

We prove the solvability of (1.12) by constructing a continuous operator $\mathfrak{R}$ in $C^{\alpha^{\prime}, 0}\left(S_{T}\right)$ (the regularizer) such that

$$
\mathfrak{A} \mathfrak{R} g=(I+\mathfrak{T}) g, \quad g \in C^{\alpha^{\prime}, 0}\left(S_{T}\right)
$$

where $\mathfrak{T}$ is also a continuous operator and $I+\mathfrak{T}$ is invertible. Then $m_{0}=\mathfrak{R}(I+\mathfrak{T})^{-1} g$ is a solution of (1.12).

Let $\left\{\varphi_{k}(x)\right\}, k=1, \ldots, M$, be a sufficiently "fine" smooth partition of unity on $S$ (i.e., $\left.1=\sum_{k} \varphi_{k}(x)\right)$ subordinate to the covering of $S$ with the sets $U_{k}(\delta)=\{y \in S$ : $\left.\left|x_{k}-y\right| \leq \delta \ll 1\right\}$ such that, for an arbitrarily small $\delta$, at most $M_{1}$ sets $U_{k}(\delta)$ can have nonempty intersection at every point $x \in S$. Moreover, let $\varphi_{k}(x), \chi_{k}(x)$, and $\omega_{k}(x)$ be functions with the following properties:

$$
\begin{gathered}
\psi_{k}(x) \varphi_{k}(x)=\varphi_{k}(x), \quad \chi_{k}(x) \psi_{k}(x)=\psi_{k}(x), \quad \omega_{k}(x) \chi_{k}(x)=\chi_{k}(x), \\
0 \leq \varphi_{k}, \psi_{k}, \chi_{k}, \omega_{k} \leq 1, \\
\operatorname{supp} \psi_{k}, \operatorname{supp} \chi_{k}, \operatorname{supp} \omega_{k} \subset U_{k}(2 \delta), \operatorname{supp} \varphi_{k} \subset U_{k}(\delta), \\
\left|\nabla \varphi_{k}\right|+\left|\nabla \psi_{k}\right|+\left|\nabla \chi_{k}\right|+\left|\nabla \omega_{k}\right| \leq c \delta^{-1}
\end{gathered}
$$


We assume that in a neighborhood of $x_{k}$ the surface $S$ is given by the equation

$$
y_{3}^{(k)}=\Phi_{k}\left(y^{\prime(k)}\right), \quad y^{(k)}=\left(y_{1}^{(k)}, y_{2}^{(k)}\right),
$$

where the $\left\{y_{j}^{(k)}\right\}$ are local Cartesian coordinates at the point $x_{k}$, the $y_{3}^{(k)}$-axis being directed along the interior normal $-\boldsymbol{n}\left(x_{k}\right)$ to $S$. The function $\Phi_{k}$ is defined in a disk $D=\left\{\left|y^{\prime(k)}\right| \leq d\right\}$ with $d>4 \delta$ (these numbers are independent of $k$ ) and belongs to $C^{1+\alpha}(D)$. The norms of $\Phi_{k}$ are bounded uniformly in $k$, moreover,

$$
\left|\Phi_{k}\left(y^{(k)}\right)\right| \leq c\left|y^{\prime(k)}\right|^{1+\alpha}, \quad\left|\nabla \Phi_{k}\left(y^{\prime(k)}\right)\right| \leq c\left|y^{\prime(k)}\right|^{\alpha}, \quad y^{\prime(k)} \in D .
$$

The coordinates $\left\{y_{j}^{(k)}\right\}$ are related to $\{x\}$ by

$$
y^{(k)}=C_{k}\left(x-x_{k}\right)
$$

with an orthogonal matrix $C_{k}$. This transformation establishes one-to-one correspondence between the set $\mathcal{U}_{k}=\left\{y_{3}^{(k)}=\Phi_{k}\left({y^{\prime}}^{(k)}\right), y^{\prime k} \in D\right\}$ and a certain neighborhood $U_{k} \equiv U_{k}(d) \subset S$ of $x_{k}$. We often write $\{y\}$ instead of $\left\{y^{(k)}\right\}$.

Let $\mathfrak{A}_{k}$ be the operator $\mathfrak{A}_{0}=-2 \mu_{1} \mathfrak{G}_{0} \mathfrak{S}_{0}+\frac{\mu_{2}}{2} I$ computed on the tangential plane $\mathbb{T}_{k}$ to $S$ at the point $x_{k}$. The operator $\mathfrak{R}_{k}=\mathfrak{A}_{k}^{-1}$ is given by (1.17) (in the coordinates $\left\{y^{\prime}\right\}$ ):

$$
\begin{aligned}
\mathfrak{R}_{k} g_{k} & =\frac{2}{\mu_{2}} g_{k}\left(y^{\prime}, t\right)-\frac{2 \mu_{1}}{\mu_{2}} \int_{0}^{t} d \tau \int_{\mathbb{R}^{2}} L\left(y^{\prime}-\eta^{\prime}, t-\tau\right) g_{k}\left(\eta^{\prime}, \tau\right) d \eta^{\prime} \\
& =\frac{2}{\mu_{2}} g_{k}\left(y^{\prime}, t\right)-\frac{2 \mu_{1}}{\mu_{2}} \mathfrak{L}_{k} g_{k},
\end{aligned}
$$

where $g_{k}\left(y^{\prime}, t\right)$ is the function $\varphi_{k} g$ written in the coordinates $y^{\prime}$. We set

$$
\mathfrak{R} g=\sum_{k=1}^{M} \psi_{k} \mathfrak{R}_{k} \varphi_{k} g .
$$

Then

$$
\begin{aligned}
\mathfrak{A} \mathfrak{R} g=\sum_{k} \mathfrak{A} \psi_{k} \mathfrak{R}_{k} g_{k} & =\sum_{k}\left(\mathfrak{A} \chi_{k}-\chi_{k} \mathfrak{A}\right) \psi_{k} \mathfrak{R}_{k} g_{k}+\sum_{k} \chi_{k}\left(\mathfrak{A}-\mathfrak{A}_{k}\right) \psi_{k} \mathfrak{R}_{k} g_{k} \\
& +\sum_{k} \chi_{k} \mathfrak{A}_{k}\left(\psi_{k} \mathfrak{R}_{k}-\mathfrak{R}_{k} \psi_{k}\right) g_{k}+g, \quad g_{k}=g \varphi_{k},
\end{aligned}
$$

whence

$$
\begin{aligned}
& \mathfrak{T} g=\sum_{k}\left(\mathfrak{A} \chi_{k}-\chi_{k} \mathfrak{A}\right) \psi_{k} \mathfrak{R}_{k} g_{k}+\sum_{k} \chi_{k}\left(\mathfrak{A}-\mathfrak{A}_{k}\right) \psi_{k} \mathfrak{R}_{k} g_{k} \\
&+\sum_{k} \chi_{k} \mathfrak{A}_{k}\left(\psi_{k} \mathfrak{R}_{k}-\mathfrak{R}_{k} \psi_{k}\right) g_{k} \\
& \equiv \sum_{k}\left(\mathfrak{T}_{k}^{(1)} g_{k}+\mathfrak{T}_{k}^{(2)} g_{k}+\mathfrak{T}_{k}^{(3)} g_{k}\right)=\sum_{k=1}^{M} \mathfrak{T}_{k} g_{k} .
\end{aligned}
$$

The proof of the invertibility of $I+\mathfrak{T}$ can be outlined as follows. We introduce a new norm in $C^{\alpha^{\prime}, 0}\left(S_{T}\right)$ :

$$
\|u\|_{C^{\alpha^{\prime}, 0}\left(S_{T}\right)}=\max _{m}\left\|\varphi_{m} u\right\|_{C^{\alpha^{\prime}, 0}\left(U_{m, T}\right)},
$$


where $U_{m, T}=U_{m}(d) \times(0, T)$. It will be shown that

$$
\begin{gathered}
\sup _{S_{t}}\left|\mathfrak{T}_{k} g_{k}\right| \leq c(\delta) \int_{0}^{t}\left\|g_{k}\right\|_{C^{\alpha^{\prime}, 0}\left(U_{k, \tau}\right)} \frac{d \tau}{(t-\tau)^{1-\epsilon / 2}}, \\
\left\|\mathfrak{T}_{k} g_{k}\right\|_{C^{\alpha^{\prime}, 0}\left(U_{k, t}\right)} \leq c_{0} \delta\left\|g_{k}\right\|_{C^{\alpha^{\prime}, 0}\left(U_{k, t}\right)}+c(\delta) \int_{0}^{t}\left\|g_{k}\right\|_{C^{\alpha^{\prime}, 0}\left(U_{k, \tau}\right)} \frac{d \tau}{(t-\tau)^{1-\epsilon / 2}}
\end{gathered}
$$

with $0<\epsilon<1$ and with a small $\delta>0$ (in what follows we do not distinguish exact values of the parameter $\epsilon$ from estimates (1.32), (1.33), etc. and take as $\epsilon$ the minimal value of these parameters). The constant $c_{0}$ is independent of $\delta$. Since only a fixed number $M_{1}$ of the sets $U_{k}$ can have nonempty intersection at every point, we have

$$
\begin{aligned}
& \left\|\varphi_{m} \mathfrak{T} g\right\|_{C^{\alpha^{\prime}, 0}\left(U_{m, t}\right)} \leq\|\mathfrak{T} g\|_{C^{\alpha^{\prime}, 0}\left(U_{m, t}\right)}+\left\|\varphi_{m}\right\|_{C^{\alpha^{\prime}\left(U_{m}\right)}} \sup _{U_{m, t}}|\mathfrak{T} g(x, \tau)| \\
& \leq c \max _{k}\left(\left\|\mathfrak{T}_{k} g_{k}\right\|_{C^{\alpha^{\prime}, 0}\left(U_{k, t}\right)}+c(\delta) \sup _{U_{k, t}}\left|\mathfrak{T}_{k} g_{k}(x, t)\right|\right) \\
& \leq \max _{k}\left(c_{0} \delta\left\|g_{k}\right\|_{C^{\alpha^{\prime}, 0}\left(U_{k, t}\right)}+c(\delta) \int_{0}^{t}\left\|g_{k}\right\|_{C^{\alpha^{\prime}, 0}\left(U_{k, \tau}\right)} \frac{d \tau}{(t-\tau)^{1-\epsilon / 2}}\right) .
\end{aligned}
$$

It follows that

$$
\|\mathfrak{T} g\|_{C^{\alpha^{\prime}, 0}\left(S_{t}\right)} \leq c_{1} \delta\|g\|_{C^{\alpha^{\prime}, 0}\left(S_{t}\right)}+c(\delta) \int_{0}^{t}\|g\|_{C^{\alpha^{\prime}, 0}\left(S_{\tau}\right)} \frac{d \tau}{(t-\tau)^{1-\epsilon / 2}} .
$$

If $c_{1} \delta<1$, then (3.9) implies the invertibility of $I+\mathfrak{T}$. Indeed, let us solve the equation $(I+\mathfrak{T}) g=h$ by iteration:

$$
g_{m+1}=h-\mathfrak{T} g_{m}, \quad g_{0}=0 .
$$

The differences $\mathfrak{g}_{m+1}=g_{m+1}-g_{m}$ satisfy

$$
\mathfrak{g}_{m+1}=-\mathfrak{T} \mathfrak{g}_{m}, \quad \mathfrak{g}_{1}=h .
$$

By (3.9),

$$
x_{m+1}(t) \leq c_{1} \delta x_{m}(t)+c(\delta) \int_{0}^{t} x_{m}(\tau) \frac{d \tau}{(t-\tau)^{1-\epsilon / 2}},
$$

where $x_{m}(t)=\left\|\mathfrak{g}_{m}\right\|_{C^{\alpha^{\prime}, 0}\left(S_{t}\right)}$. Hence, $X_{q}(t)=\sum_{m=1}^{q} x_{m}(t)$ satisfies

$$
X_{q+1}(t) \leq X_{1}(t)+c_{1} \delta X_{q}(t)+c(\delta) \int_{0}^{t} X_{q}(\tau) \frac{d \tau}{(t-\tau)^{1-\epsilon / 2}} .
$$

It follows that

$$
X_{q+1}(t) \leq \frac{x_{1}(t)}{1-c_{1} \delta}+\frac{c(\delta)}{1-c_{1} \delta} \int_{0}^{t} X_{q}(\tau) \frac{d \tau}{(t-\tau)^{1-\epsilon / 2}} .
$$

Arguing as in the proof of (2.12), we obtain a uniform (with respect to $q$ ) estimate for $X_{q}(t)$ :

$$
X_{q}(t) \leq c(T) x_{1}(t) \leq c(T)\|h\|_{C^{\alpha^{\prime}, 0}\left(S_{t}\right)}
$$

which implies the convergence of $g_{m}$ to the solution of $(I+\mathfrak{T}) g=h$. and the estimate

$$
(I+\mathfrak{T})^{-1} \leq c(T) .
$$

Returning to formulas $(3.5),(3.6)$, we consider the expressions $\mathfrak{T}_{k}^{(j)} g_{k}, j=1,3$. To prove inequalities (3.8) for these expressions, we need some auxiliary propositions.

Proposition 4. If $\psi \in C^{\beta}(S)$ with $\beta \in\left(\alpha^{\prime}, 1\right)$, then

$$
\|(\mathfrak{S} \psi-\psi \mathfrak{S}) h\|_{C^{\alpha^{\prime}}(S)} \leq c \sup _{S}|h(y)|
$$

with a constant proportional to the norm of $\psi$. 
Proof. The function $\boldsymbol{u}=(\mathfrak{S} \psi-\psi \mathfrak{S}) h$ is given by

$$
\boldsymbol{u}(x)=\int_{S} \mathcal{E}(x, y) h(y) d S
$$

with $\mathcal{E}(x, y)=\boldsymbol{n}(x) \times \nabla E(x-y)(\psi(x)-\psi(y))$. We have

$$
\begin{aligned}
|\boldsymbol{u}(x)| & \leq \sup _{S}|h(x)|\|\psi\|_{C^{\beta}(S)} \int_{S} \frac{d S}{|x-y|^{2-\beta}} \leq c \sup _{S}|h(x)|, \\
\boldsymbol{u}(x)-\boldsymbol{u}(z) & =\int_{\sigma_{r}} \mathcal{E}(x, y) h(y) d S-\int_{\sigma_{r}} \mathcal{E}(z, y) h(y) d S \\
& +\int_{S \backslash \sigma_{r}}(\mathcal{E}(x, y)-\mathcal{E}(z, y)) h(y) d S \equiv I_{1}+I_{2}+I_{3}, \quad x, z \in S,
\end{aligned}
$$

where $r=|x-z|$ and $\sigma_{r}=\{y \in S:|y-x| \leq 2 r\}$. Clearly,

$$
\left|I_{1}\right|+\left|I_{2}\right| \leq c \sup _{S}|h(y)|\|\psi\|_{C^{\beta}(S)} r^{\beta},
$$

and moreover, since

$$
\begin{aligned}
\mathcal{E}(x, y)-\mathcal{E}(z, y)=(\boldsymbol{n}(x)-\boldsymbol{n}(z)) \times \nabla E(x-y)(\psi(x)-\psi(y))+(\psi(x)-\psi(z)) \boldsymbol{n}(z) \\
\times \nabla E(x-y)+\boldsymbol{n}(z) \times(\nabla E(x-y)-\nabla E(z-y))(\psi(z)-\psi(y)),
\end{aligned}
$$

we have

$$
\begin{aligned}
\left|I_{3}\right| \leq c\left(r^{\alpha} \sup _{S}|h(y)|\|\psi\|_{C^{\beta}(S)}+r^{\beta}\|\psi\|_{C^{\beta}(S)} \sup _{S}|h(y)| \int_{S \backslash \sigma_{r}} \frac{d S}{|x-y|^{2}}\right. \\
\left.+r\|\psi\|_{C^{\beta}(S)} \sup _{S}|h(y)| \int_{S \backslash \sigma_{r}} \frac{d S}{|x-y|^{3-\beta}}\right) \leq c r^{\alpha^{\prime}} \sup _{S}|h(y)|\|\psi\|_{C^{\beta}(S)} .
\end{aligned}
$$

Collecting the estimates, we obtain (3.10).

Proposition 5. If $\psi \in C^{\beta}(S)$ with $\beta \in\left(\alpha^{\prime}, 1\right)$, then

$$
\|(\mathfrak{G} \psi-\psi \mathfrak{G}) \boldsymbol{h}\|_{C^{\alpha^{\prime}, 0}\left(S_{t}\right)} \leq c \int_{0}^{t} \sup _{S_{\tau}}|\boldsymbol{h}(y, \tau)| \frac{d \tau}{(t-\tau)^{1-\epsilon / 2}}
$$

with $\epsilon>0$ and with the constant c proportional to the norm of $\psi$.

Proof. The proof is similar to that of Proposition 4. We have

$$
u(x, t) \equiv(\mathfrak{G} \psi-\psi \mathfrak{G}) \boldsymbol{h}=\int_{0}^{t} d \tau \int_{S} \boldsymbol{G}(x, y, t-\tau) \cdot \boldsymbol{h}(y, \tau) d S,
$$

where $\boldsymbol{G}(x, y, t-\tau)=\boldsymbol{n}(x) \times \nabla \Gamma(x-y, t-\tau)(\psi(x)-\psi(y))$. Hence,

$$
\begin{aligned}
|u(x, t)| & \leq c\|\psi\|_{C^{\beta}(S)} \int_{0}^{t} \sup _{S}|\boldsymbol{h}(y, \tau)| d \tau \int_{S} \frac{d S}{\left(|x-y|^{2}+t-\tau\right)^{2-\beta / 2}} \\
& \leq c \int_{0}^{t} \sup _{S}|\boldsymbol{h}(y, \tau)| \frac{d \tau}{(t-\tau)^{1-\beta / 2}}
\end{aligned}
$$

$$
\begin{aligned}
u(x, t) & -u(z, t) \\
= & \int_{0}^{t} d \tau \int_{\sigma_{r}} \boldsymbol{G}(x, y, t-\tau) \cdot \boldsymbol{h}(y, \tau) d S-\int_{0}^{t} d \tau \int_{\sigma_{r}} \boldsymbol{G}(z, y, t-\tau) \cdot \boldsymbol{h}(y, \tau) d S \\
& +\int_{0}^{t} d \tau \int_{S \backslash \sigma_{r}}(\boldsymbol{G}(x, y, \tau)-\boldsymbol{G}(z, y, t-\tau)) \cdot \boldsymbol{h}(y, \tau) d S \equiv J_{1}+J_{2}+J_{3},
\end{aligned}
$$


and

$$
\left|J_{1}\right|+\left|J_{2}\right| \leq c \int_{0}^{t} \sup _{S}|\boldsymbol{h}(y, \tau)| \frac{d \tau}{(t-\tau)^{1-\beta / 2}}
$$

$$
\begin{aligned}
& \boldsymbol{G}(x, y, t-\tau)-\boldsymbol{G}(z, y, t-\tau) \\
& \begin{array}{r}
=(\boldsymbol{n}(x)-\boldsymbol{n}(z)) \times \nabla \Gamma(x-y, t-\tau)(\psi(x)-\psi(y))+(\psi(x)-\psi(z)) \boldsymbol{n}(z) \times \nabla \Gamma(x-y, t-\tau) \\
+\boldsymbol{n}(z) \times(\nabla \Gamma(x-y, t-\tau)-\nabla \Gamma(z-y, t-\tau))(\psi(z)-\psi(y)),
\end{array}
\end{aligned}
$$

which implies

$$
\begin{gathered}
\left|\int_{0}^{t} d \tau \int_{S \backslash \sigma_{r}}(\boldsymbol{G}(x, y, t-\tau)-\boldsymbol{G}(z, y, t-\tau)) \cdot \boldsymbol{h}(y, \tau) d S\right| \\
\leq c\left(r^{\alpha} \int_{0}^{t} \sup _{S}|\boldsymbol{h}(y, \tau)| \frac{d \tau}{(t-\tau)^{1-\beta / 2}}+r^{\beta} \int_{0}^{t} \sup _{S}|\boldsymbol{h}(y, \tau)| d \tau \int_{S \backslash \sigma_{r}} \frac{d S}{\left(|x-y|^{2}+t-\tau\right)^{2}}\right. \\
\left.\quad+r \int_{0}^{t} \sup _{S}|\boldsymbol{h}(y, \tau)| d \tau \int_{S \backslash \sigma_{r}} \frac{d S}{\left(|x-y|^{2}+t-\tau\right)^{5 / 2-\beta / 2}}\right) \\
\leq c r^{\alpha^{\prime}} \int_{0}^{t} \sup _{S}|\boldsymbol{h}(y, \tau)| \frac{d \tau}{(t-\tau)^{1-\epsilon / 2}} .
\end{gathered}
$$

Collecting the estimates, we arrive at (3.12).

A similar estimate is true for

$$
\left(\mathfrak{R}_{k} \psi-\psi \mathfrak{R}_{k}\right) j=\int_{0}^{t} d \tau \int_{\mathbb{R}^{2}} L\left(x^{\prime}-y^{\prime}, t-\tau\right)\left(\psi\left(x^{\prime}\right)-\psi\left(y^{\prime}\right)\right) j\left(y^{\prime}, \tau\right) d y^{\prime} \equiv v\left(x^{\prime}, t\right),
$$

where $L$ is a kernel satisfying (1.18).

Proposition 6. If $\psi \in C^{\beta, 0}\left(\mathbb{R}_{t}^{2}\right)$ and $\alpha^{\prime} \in(0, \beta)$, then

$$
\|v\|_{C^{\alpha^{\prime}, 0}\left(\mathbb{R}_{t}^{2}\right)} \leq c \int_{0}^{t} \sup _{\mathbb{R}^{2}}\left|j\left(y^{\prime}, \tau\right)\right| \frac{d \tau}{(t-\tau)^{1-\epsilon / 2}}, \quad \epsilon>0 .
$$

The proof is the same as in the preceding proposition.

We proceed by estimating the expressions $\mathfrak{T}_{k}^{(1)} g_{k}=\left(\mathfrak{A} \chi_{k}-\chi_{k} \mathfrak{A}\right) \psi_{k} \mathfrak{R} g_{k}$ and $\mathfrak{T}_{k}^{(3)} g_{k}=$ $\psi_{k} \mathfrak{A}_{k}\left(\psi_{k} \mathfrak{R}_{k}-\mathfrak{R}_{k} \psi_{k}\right) g_{k}$. We make use of the formula

$$
\mathfrak{A} \chi_{k}-\chi_{k} \mathfrak{A}=-2 \mu_{1}\left(\mathfrak{G} \chi_{k}-\chi_{k} \mathfrak{G}\right) \mathfrak{S}-2 \mu_{1} \mathfrak{G}\left(\mathfrak{S} \chi_{k}-\chi_{k} \mathfrak{S}\right) .
$$

By (1.32), (3.10), (3.12), and (3.14), we have

$$
\begin{aligned}
& \left\|\mathfrak{G}\left(\mathfrak{S} \chi_{k}-\chi_{k} \mathfrak{S}\right) \psi_{k} \mathfrak{R}_{k} g_{k}\right\|_{C^{\alpha^{\prime}, 0}\left(S_{t}\right)} \\
& \quad \leq c \int_{0}^{t}\left\|\left(\mathfrak{S} \chi_{k}-\chi_{k} \mathfrak{S}\right) \psi_{k} \mathfrak{R}_{k} g_{k}\right\|_{C^{\alpha^{\prime \prime}}(S)} \frac{d \tau}{(t-\tau)^{1-\epsilon / 2}} \\
& \quad \leq c \int_{0}^{t} \sup _{U_{k, \tau}}\left|\psi_{k} \mathfrak{R}_{k} g_{k}\right| \frac{d \tau}{(t-\tau)^{1-\epsilon / 2}} \leq c \int_{0}^{t}\left\|g_{k}\right\|_{C^{\alpha^{\prime}, 0}\left(U_{k, \tau}\right)} \frac{d \tau}{(t-\tau)^{1-\epsilon / 2}}
\end{aligned}
$$

$$
\begin{aligned}
& \left\|\left(\mathfrak{G} \chi_{k}-\chi_{k} \mathfrak{G}\right) \mathfrak{S} \psi_{k} \mathfrak{R}_{k} g_{k}\right\|_{C^{\alpha^{\prime}, 0}\left(S_{t}\right)} \leq c \int_{0}^{t} \max _{S_{\tau}}\left|\mathfrak{S} \psi_{k} \mathfrak{R}_{k} g_{k}\right| \frac{d \tau}{(t-\tau)^{1-\epsilon / 2}} \\
& \quad \leq c \int_{0}^{t}\left\|g_{k}\right\|_{C^{\alpha^{\prime}, 0}\left(U_{k, \tau}\right)} \frac{d \tau}{(t-\tau)^{1-\epsilon / 2}}, \\
& \left\|\psi_{k} \mathfrak{A}_{k}\left(\psi_{k} \mathfrak{R}_{k}-\mathfrak{R}_{k} \psi_{k}\right) g_{k}\right\|_{C^{\alpha^{\prime}, 0}\left(U_{k, t}\right)} \leq c\left\|\left(\psi_{k} \mathfrak{R}_{k}-\mathfrak{R}_{k} \psi_{k}\right) g_{k}\right\|_{C^{\alpha^{\prime}, 0}\left(U_{k, t}\right)} \\
& \leq c \int_{0}^{t} \sup _{U_{k, \tau}}\left|g_{k}\right| \frac{d \tau}{(t-\tau)^{1-\epsilon / 2}}, \quad \epsilon \in(0,1) .
\end{aligned}
$$


Hence,

$$
\left\|\mathfrak{T}_{k}^{(1)} g_{k}\right\|_{C^{\alpha^{\prime}, 0}\left(U_{k, t}\right)}+\left\|\mathfrak{T}_{k}^{(3)} g_{k}\right\|_{C^{\alpha^{\prime}, 0}\left(U_{k, t}\right)} \leq c \int_{0}^{t}\left\|g_{k}\right\|_{C^{\alpha^{\prime}, 0}\left(U_{k, \tau}\right)} \frac{d \tau}{(t-\tau)^{1-\epsilon / 2}} .
$$

Finally, we turn our attention to $\mathfrak{T}^{(2)} \mathrm{g}$. We show that the expressions $\chi_{k}\left(\mathfrak{A}-\mathfrak{A}_{k}\right) \psi_{k}$ can be made meaningful, although the operators $\mathfrak{A}$ and $\mathfrak{A}_{k}$ are defined in different spaces. We work in the coordinates $\left\{y^{(k)}\right\}$. Since

$$
\mathfrak{A}=-2 \mu_{1} \mathfrak{G} \mathfrak{S}+\frac{\mu_{2}}{2} I, \quad \mathfrak{A}_{k}=-2 \mu_{1} \mathfrak{G}_{k} \mathfrak{S}_{k}+\frac{\mu_{2}}{2} I,
$$

where $\mathfrak{G}_{k}$ and $\mathfrak{S}_{k}$ are the operators $\mathfrak{G}_{0}, \mathfrak{S}_{0}$ defined on the plane $\mathbb{T}_{k}$, we have

$$
\begin{aligned}
\chi_{k}\left(\mathfrak{A}-\mathfrak{A}_{k}\right) \psi_{k}= & -2 \mu_{1} \chi_{k}\left(\mathfrak{G} \mathfrak{S}-\mathfrak{G}_{k} \mathfrak{S}_{k}\right) \psi_{k}=-2 \mu_{1} \chi_{k}\left(\mathfrak{G} \omega_{k} \mathfrak{S}-\mathfrak{G}_{k} \omega_{k} \mathfrak{S}_{k}\right) \psi_{k} \\
& -2 \mu_{1} \chi_{k}\left(\mathfrak{G}_{k} \omega_{k}-\omega_{k} \mathfrak{G}_{k}\right) \mathfrak{S}_{k} \psi_{k}+2 \mu_{1} \chi_{k}\left(\mathfrak{G} \omega_{k}-\omega_{k} \mathfrak{G}\right) \mathfrak{S} \psi_{k} .
\end{aligned}
$$

We represent $\mathfrak{S}$ and $\mathfrak{G}$ in the form $\mathfrak{S}=\mathfrak{S}^{\prime}+\mathfrak{S}^{\prime \prime}, \mathfrak{G}=\mathfrak{G}^{\prime}+\mathfrak{G}^{\prime \prime}$, where

$$
\begin{aligned}
\mathfrak{S}^{\prime \prime} m & =\int_{S}(\boldsymbol{n}(x)-\boldsymbol{n}(y)) \times \nabla E(x-y) m(y, t) d S, \\
\mathfrak{S}^{\prime} m & =\int_{S} \boldsymbol{n}(y) \times \nabla E(x-y) m(y, t) d S, \\
\mathfrak{G}^{\prime \prime} \boldsymbol{\lambda} & =\int_{0}^{t} d \tau \int_{S}(\boldsymbol{n}(x)-\boldsymbol{n}(y)) \times \nabla \Gamma(x-y, t-\tau) \cdot \boldsymbol{\lambda}(y, t) d S, \\
\mathfrak{G}^{\prime} \boldsymbol{\lambda} & =\int_{0}^{t} d \tau \int_{S} \boldsymbol{n}(y) \times \nabla \Gamma(x-y, t-\tau) \cdot \boldsymbol{\lambda}(y, t) d S .
\end{aligned}
$$

Next, we extend $\Phi_{k}\left(y^{\prime k}\right)$ from the disk $D_{2 \delta}=\left\{\left|y^{\prime}\right| \leq 2 \delta\right\}$ into $\mathbb{R}^{2}$ in such a way that the extended function $\Phi_{k}^{*}$ vanishes for $\left|y^{\prime k}\right| \geq 4 \delta$ and satisfies (3.3). We denote by $S_{k}^{*}$ the surface given by

$$
y_{3}^{(k)}=\Phi_{k}^{*}\left(y^{\prime k}\right), \quad y^{\prime k} \in \mathbb{R}^{2} .
$$

Finally, we set

$$
\begin{aligned}
\mathfrak{S}_{k}^{*} m & =\int_{S_{k}^{*}} \boldsymbol{n}^{*}(y) \times \nabla E(x-y) m(y, t) d S, \\
\mathfrak{G}_{k}^{*} \boldsymbol{\lambda} & =\int_{0}^{t} d \tau \int_{S_{k}^{*}} \boldsymbol{n}^{*}(y) \times \nabla \Gamma(x-y, t-\tau) \cdot \boldsymbol{\lambda}(y, t) d S .
\end{aligned}
$$

By $\boldsymbol{n}^{*}$ we mean the normal to $S_{k}^{*}$ (it is equal to $\boldsymbol{e}_{3}$ for $\left|y^{\prime}\right| \geq 4 \delta$ ).

Since supp $\chi_{k}$, supp $\psi_{k}$, supp $\omega_{k} \subset U_{k}(2 \delta)$, we have

$$
\chi_{k} \mathfrak{G}^{\prime} \omega_{k}=\chi_{k} \mathfrak{G}_{k}^{*} \omega_{k}, \quad \omega_{k} \mathfrak{S}^{\prime} \psi_{k}=\omega_{k} \mathfrak{S}_{k}^{*} \psi_{k},
$$

so that

$$
\begin{aligned}
\chi_{k}( & \left.\mathfrak{G} \omega_{k} \mathfrak{S}-\mathfrak{G}_{k} \omega_{k} \mathfrak{S}_{k}\right) \psi_{k} \\
= & \chi_{k}\left(\mathfrak{G}^{\prime \prime} \omega_{k} \mathfrak{S}+\mathfrak{G}^{\prime} \omega_{k} \mathfrak{S}^{\prime \prime}\right) \psi_{k}+\chi_{k}\left(\mathfrak{G}_{k}^{*} \omega_{k} \mathfrak{S}_{k}^{*}-\mathfrak{G}_{k} \omega_{k} \mathfrak{S}_{k}\right) \psi_{k} \\
= & \chi_{k}\left(\left(\mathfrak{G}_{k}^{*}-\mathfrak{G}_{k}\right)\left(\mathfrak{S}_{k}^{*}-\mathfrak{S}_{k}\right)+\left(\mathfrak{G}_{k}^{*}-\mathfrak{G}_{k}\right) \mathfrak{S}_{k}+\mathfrak{G}_{k}\left(\mathfrak{S}_{k}^{*}-\mathfrak{S}_{k}\right)\right) \psi_{k} \\
& +\chi_{k}\left(\left(\left(\mathfrak{G}_{k}^{*}-\mathfrak{G}_{k}\right) \omega_{k}-\omega_{k}\left(\mathfrak{G}_{k}^{*}-\mathfrak{G}_{k}\right)\right)\left(\mathfrak{S}_{k}^{*}-\mathfrak{S}_{k}\right)+\left(\left(\mathfrak{G}_{k}^{*}-\mathfrak{G}_{k}\right) \omega_{k}-\omega_{k}\left(\mathfrak{G}_{k}^{*}-\mathfrak{G}_{k}\right)\right) \mathfrak{S}_{k}\right. \\
& \left.+\mathfrak{G}_{k}\left(\omega_{k}\left(\mathfrak{S}_{k}^{*}-\mathfrak{S}_{k}\right)-\left(\mathfrak{S}_{k}^{*}-\mathfrak{S}_{k}\right) \omega_{k}\right)+\chi_{k}\left(\mathfrak{G}^{\prime \prime} \omega_{k} \mathfrak{S}+\mathfrak{G}^{\prime} \omega_{k} \mathfrak{S}^{\prime \prime}\right)\right) \psi_{k}
\end{aligned}
$$

and

$$
\mathfrak{T}^{(2)} g=\mathfrak{T}^{(4)} g+\mathfrak{T}^{(5)} g,
$$


where

$(3.20) \mathfrak{T}^{(4)} g=-2 \mu_{1} \sum_{k=1}^{M} \chi_{k}\left(\left(\mathfrak{G}_{k}^{*}-\mathfrak{G}_{k}\right)\left(\mathfrak{S}_{k}^{*}-\mathfrak{S}_{k}\right)+\left(\mathfrak{G}_{k}^{*}-\mathfrak{G}_{k}\right) \mathfrak{S}_{k}+\mathfrak{G}_{k}\left(\mathfrak{S}_{k}^{*}-\mathfrak{S}_{k}\right)\right) \psi_{k} \mathfrak{R}_{k} g_{k}$ is the principal part of $\mathfrak{T}^{(2)} \mathrm{g}$, and

$$
\begin{aligned}
& \left.\mathfrak{T}^{(5)} g=-2 \mu_{1} \sum_{k=1}^{M} \chi_{k}\left(\mathfrak{G}^{\prime \prime} \omega_{k} \mathfrak{S}+\mathfrak{G}^{\prime} \omega_{k} \mathfrak{S}^{\prime \prime}\right)\right) \psi_{k} \mathfrak{R}_{k} g_{k} \\
& \begin{aligned}
-2 \mu_{1} \sum_{k=1}^{M} \chi_{k}\left(\left(\left(\mathfrak{G}_{k}^{*}-\mathfrak{G}_{k}\right) \omega_{k}-\omega_{k}\left(\mathfrak{G}_{k}^{*}\right.\right.\right. & \left.\left.-\mathfrak{G}_{k}\right)\right) \mathfrak{S}_{k}^{*}+\mathfrak{G}_{k}\left(\omega_{k}\left(\mathfrak{S}_{k}^{*}-\mathfrak{S}_{k}\right)-\left(\mathfrak{S}_{k}^{*}-\mathfrak{S}_{k}\right) \omega_{k}\right) \\
& \left.+\left(\mathfrak{G}_{k} \omega_{k}-\omega_{k} \mathfrak{G}_{k}\right) \mathfrak{S}_{k}-\left(\mathfrak{G} \omega_{k}-\omega_{k} \mathfrak{G}\right) \mathfrak{S}\right) \psi_{k} \mathfrak{R}_{k} g_{k}
\end{aligned}
\end{aligned}
$$

is the sum of the remaining terms in $\mathfrak{T}^{(2)} g$, such that

$$
\left\|\mathfrak{T}^{(5)} g\right\|_{C^{\alpha^{\prime}, 0}\left(S_{t}\right)} \leq c \int_{0}^{t}\|\boldsymbol{g}\|_{C^{\alpha^{\prime}, 0}\left(S_{\tau}\right.} \frac{d \tau}{(t-\tau)^{1-\epsilon / 2}}, \quad \epsilon \in(0,1),
$$

in view of Propositions 4, 5, and 6 .

We consider $\mathfrak{T}^{(4)} g$. In the coordinate system $\left\{y^{(k)}\right\}$, we have

$$
\begin{aligned}
\left(\mathfrak{S}_{k}^{*}-\mathfrak{S}_{k}\right) f & =\int_{\mathbb{R}^{2}}\left(\tilde{\boldsymbol{n}}\left(\eta^{\prime}\right) \times \nabla E(y-\eta)-\boldsymbol{e}_{3} \cdot \nabla E\left(y^{\prime}-\eta^{\prime}, 0\right)\right) f\left(\eta^{\prime}, t\right) d \eta^{\prime}, \\
\left(\mathfrak{G}_{k}^{*}-\mathfrak{G}_{k}\right) f=\int_{0}^{t} d \tau \int_{\mathbb{R}^{2}}\left(\left(\widetilde{\boldsymbol{n}}\left(\eta^{\prime}\right) \times \nabla \Gamma(y-\eta, t-\tau)\right.\right. & \left.\quad-\boldsymbol{e}_{3} \times \nabla \Gamma\left(y^{\prime}-\eta^{\prime}, 0, t-\tau\right)\right) f\left(\eta^{\prime}, \tau\right) d \eta^{\prime}
\end{aligned}
$$

where

$$
\widetilde{\boldsymbol{n}}\left(\eta^{\prime}\right)=\boldsymbol{n}^{*}\left(\eta^{\prime}\right) \sqrt{1+\left|\nabla^{\prime} \Phi_{k}^{*}\left(\eta^{\prime}\right)\right|^{2}}=\left(-\Phi_{k, \eta_{1}}^{*},-\Phi_{k, \eta_{2}}^{*}, 1\right)
$$

and

$$
y-\eta=\left(y^{\prime}-\eta^{\prime}, \Phi_{k}^{*}\left(y^{\prime}\right)-\Phi_{k}^{*}\left(\eta^{\prime}\right)\right) .
$$

The kernels of the potentials (3.22) are

$$
\begin{aligned}
\mathbf{E}^{*}\left(y^{\prime}, \eta^{\prime}\right) & =\tilde{\boldsymbol{n}}\left(\eta^{\prime}\right) \times \nabla E(y-\eta)-\boldsymbol{e}_{3} \times \nabla E\left(y^{\prime}-\eta^{\prime}, 0\right) \\
& =\frac{1}{4 \pi}\left(-\Phi_{k, \eta_{2}}^{*} \frac{\Phi_{k}^{*}\left(y^{\prime}\right)-\Phi_{k}^{*}\left(\eta^{\prime}\right)}{|y-\eta|^{3}}-\frac{y_{2}-\eta_{2}}{|y-\eta|^{3}}+\frac{y_{2}-\eta_{2}}{\left|y^{\prime}-\eta^{\prime}\right|^{3}}, \frac{y_{1}-\eta_{1}}{|y-\eta|^{3}}\right. \\
& \left.+\Phi_{k, \eta_{1}}^{*} \frac{\Phi_{k}^{*}\left(y^{\prime}\right)-\Phi_{k}^{*}\left(\eta^{\prime}\right)}{|y-\eta|^{3}}-\frac{y_{1}-\eta_{1}}{\left|y^{\prime}-\eta^{\prime}\right|^{3}},-\Phi_{k, \eta_{1}}^{*} \frac{y_{2}-\eta_{2}}{|y-\eta|^{3}}+\Phi_{k, \eta_{2}}^{*} \frac{y_{1}-\eta_{1}}{|y-\eta|^{3}}\right), \\
\mathbf{G}^{*}\left(y^{\prime}, \eta^{\prime}, t\right. & -\tau)=\tilde{\boldsymbol{n}}\left(\eta^{\prime}\right) \times \nabla \Gamma(y-\eta, t-\tau)-\boldsymbol{e}_{3} \times \nabla \Gamma\left(y^{\prime}-\eta^{\prime}, 0, t-\tau\right) \\
& =\frac{1}{4 \pi(t-\tau)^{3 / 2}}\left(\left(\Phi_{k, \eta_{2}}^{*} \frac{\Phi_{k}^{*}\left(y^{\prime}\right)-\Phi_{k}^{*}\left(\eta^{\prime}\right)}{2(t-\tau)}+\frac{y_{2}-\eta_{2}}{2(t-\tau)}\right) e^{-\frac{|y-\eta|^{2}}{4(t-\tau)}}\right. \\
& -\frac{y_{2}-\eta_{2}}{2(t-\tau)} e^{-\frac{\left|y^{\prime}-\eta^{\prime}\right|^{2}}{4(t-\tau)}},\left(-\frac{y_{1}-\eta_{1}}{2(t-\tau)}-\Phi_{k, \eta_{1}}^{*} \frac{\Phi_{k}^{*}\left(y^{\prime}\right)-\Phi_{k}^{*}\left(\eta^{\prime}\right)}{2(t-\tau)}\right) e^{-\frac{|y-\eta|^{2}}{4(t-\tau)}} \\
& \left.+\frac{y_{1}-\eta_{1}}{2(t-\tau)} e^{-\frac{\left|y^{\prime}-\eta^{\prime}\right|^{2}}{4(t-\tau)}}+\left(\Phi_{k, \eta_{1}}^{*} \frac{y_{2}-\eta_{2}}{2(t-\tau)}-\Phi_{k, \eta_{2}}^{*} \frac{y_{1}-\eta_{1}}{2(t-\tau)}\right) e^{-\frac{|y-\eta|^{2}}{4(t-\tau)}}\right) .
\end{aligned}
$$


Since

$$
\begin{aligned}
\frac{1}{|y-\eta|^{3}}-\frac{1}{\left|y^{\prime}-\eta^{\prime}\right|^{3}} & =\int_{0}^{1} \frac{d}{d s}\left(\left(\left|y^{\prime}-\eta^{\prime}\right|^{2}+s\left(\phi_{k}\left(y^{\prime}\right)-\phi_{k}\left(\eta^{\prime}\right)\right)^{2}\right)^{-3 / 2} d s\right. \\
& =-\frac{3}{2} \int_{0}^{1} \frac{\left(\phi_{k}\left(y^{\prime}\right)-\phi_{k}\left(\eta^{\prime}\right)\right)^{2}}{\left(\left(\left|y^{\prime}-\eta^{\prime}\right|^{2}+s\left(\phi_{k}\left(y^{\prime}\right)-\phi_{k}\left(\eta^{\prime}\right)\right)^{2}\right)^{5 / 2}\right.} d s, \\
e^{-\frac{|y-\eta|^{2}}{4(t-\tau)}}-e^{-\frac{|y-\eta|^{2}}{4(t-\tau)}} & =-\frac{\left(\phi_{k}\left(y^{\prime}\right)-\phi_{k}\left(\eta^{\prime}\right)\right)^{2}}{4(t-\tau)} \int_{0}^{1} e^{-\frac{\left|y^{\prime}-\eta^{\prime}\right|^{2}+s\left(\phi_{k}\left(y^{\prime}\right)-\phi_{k}\left(\eta^{\prime}\right)^{2}\right.}{4(t-\tau)}} d s,
\end{aligned}
$$

the kernels $\mathbf{E}^{*}$ and $\mathbf{G}^{*}$ satisfy

$$
\begin{aligned}
& \left|\mathbf{E}^{*}\left(y^{\prime}, \eta^{\prime}\right)\right| \leq c \delta\left|y^{\prime}-\eta^{\prime}\right|^{-2}, \\
& \left|\mathbf{E}^{*}\left(y^{\prime}, \eta^{\prime}\right)-\mathbf{E}^{*}\left(z^{\prime}, \eta^{\prime}\right)\right| \leq c \delta\left|y^{\prime}-z^{\prime}\right|\left|y^{\prime}-\eta^{\prime}\right|^{-3}, \text { if } 2\left|y^{\prime}-z^{\prime}\right| \leq\left|y^{\prime}-\eta^{\prime}\right|, \\
& \left|\mathbf{G}^{*}\left(y^{\prime}, \eta^{\prime}, t-\tau\right)\right| \leq c \delta\left(\left|y^{\prime}-\eta^{\prime}\right|^{2}+(t-\tau)\right)^{-2}, \\
& \quad\left|\mathbf{G}^{*}\left(y^{\prime}, \eta^{\prime}, t-\tau\right)-\mathbf{G}^{*}\left(z^{\prime}, \eta^{\prime}, t-\tau\right)\right| \\
& \quad \leq c\left|y^{\prime}-z^{\prime}\right|\left(\left(\left|y^{\prime}-\eta^{\prime}\right|^{-2}+(t-\tau)\right)^{-5 / 2}\right) \text { whenever } 2\left|y^{\prime}-z^{\prime}\right| \leq\left|y^{\prime}-\eta^{\prime}\right| .
\end{aligned}
$$

In addition, from the Stokes formula it follows that

$$
\int_{\mathbb{R}^{2}} \mathbf{E}^{*}\left(y^{\prime}, \eta^{\prime}\right) d \eta^{\prime}=0, \quad \int_{\mathbb{R}^{2}} \mathbf{G}^{*}\left(y^{\prime}, \eta^{\prime}, t-\tau\right) d \eta^{\prime}=0 .
$$

We also notice that $\operatorname{supp}_{\eta} \mathbf{E}^{*}, \operatorname{supp}_{\eta} \mathbf{G}^{*} \subset \mathcal{U}_{k}(4 \delta)$.

Proposition 7. The potentials (3.22) satisfy the inequalities

$$
\begin{aligned}
\left\|\left(\mathfrak{S}_{k}^{*}-\mathfrak{S}_{k}\right) f\right\|_{C^{\alpha^{\prime}}\left(\mathbb{R}^{2}\right)} & \leq c \delta\|f\|_{C^{\alpha^{\prime}}\left(\mathbb{R}^{2}\right)}, \\
\left|\left(\mathfrak{S}_{k}^{*}-\mathfrak{S}_{k}\right) f\right| & \leq c \delta\|f\|_{C^{\alpha^{\prime}}\left(\mathbb{R}^{2}\right)}
\end{aligned}
$$

and

$$
\begin{gathered}
\left\|\left(\mathfrak{G}_{k}^{*}-\mathfrak{G}_{k}\right) \boldsymbol{f}\right\|_{C^{\alpha^{\prime}, 0}\left(\mathbb{R}^{2} \times(0, T)\right)} \leq c \delta\|\boldsymbol{f}\|_{C^{\alpha^{\prime}, 0}\left(\mathbb{R}^{2} \times(0, T)\right)}, \\
\left|\left(\mathfrak{G}_{k}^{*}-\mathfrak{G}_{k}\right) \boldsymbol{f}\right| \leq c \delta\|\boldsymbol{f}\|_{\dot{C}^{\alpha^{\prime}, 0}\left(\mathbb{R}_{T}^{2}\right)}, \\
\sup _{\mathbb{R}^{2} \times(0, t)}\left|\left(\mathfrak{G}_{k}^{*}-\mathfrak{G}_{k}\right) \boldsymbol{f}\right| \leq c \delta \int_{0}^{T}\|\boldsymbol{f}(\cdot, \tau)\|_{C^{\alpha^{\prime}\left(\mathbb{R}^{2}\right)}} \frac{d \tau}{(t-\tau)^{1-\alpha^{\prime} / 2}},
\end{gathered}
$$

where $\alpha^{\prime} \in(0, \alpha)$.

Proof. Let $\boldsymbol{u}\left(y^{\prime}\right)=\left(\mathfrak{S}_{k}^{*}-\mathfrak{S}_{k}\right) f$. We have

$$
\boldsymbol{u}\left(y^{\prime}\right)=\int_{\mathcal{U}_{k}(4 \delta)} \mathbf{E}^{*}\left(y^{\prime}, \eta^{\prime}\right)\left(f\left(\eta^{\prime}\right)-f\left(y^{\prime}\right)\right) d \eta^{\prime}=\int_{\mathbb{R}^{2}} \mathbf{E}^{*}\left(y^{\prime}, \eta^{\prime}\right)\left(f\left(\eta^{\prime}\right)-f\left(y^{\prime}\right)\right) d \eta^{\prime},
$$

which implies that

$$
\begin{aligned}
& \left|\boldsymbol{u}\left(y^{\prime}\right)\right| \leq c \delta\|f\|_{\dot{C}^{\alpha}\left(\mathbb{R}^{2}\right)}, \\
& \boldsymbol{u}\left(y^{\prime}\right)-\boldsymbol{u}\left(z^{\prime}\right) \\
& \quad=\int_{\sigma_{r}} \mathbf{E}^{*}\left(y^{\prime}, \eta^{\prime}\right)\left(f\left(\eta^{\prime}\right)-f\left(y^{\prime}\right)\right) d \eta^{\prime}-\int_{\sigma_{r}} \mathbf{E}^{*}\left(z^{\prime}, \eta^{\prime}\right)\left(f\left(\eta^{\prime}\right)-f\left(z^{\prime}\right)\right) d \eta^{\prime} \\
& \quad+\int_{\mathbb{R}^{2} \backslash \sigma_{r}}\left(\mathbf{E}^{*}\left(y^{\prime}, \eta^{\prime}\right)-\mathbf{E}^{*}\left(z^{\prime}, \eta^{\prime}\right)\right)\left(f\left(\eta^{\prime}\right)-f\left(z^{\prime}\right)\right) d \eta^{\prime}+\left(f\left(z^{\prime}\right)-f\left(y^{\prime}\right)\right) \int_{\mathbb{R}^{2} \backslash \sigma_{r}} \mathbf{E}^{*}\left(y^{\prime}, \eta^{\prime}\right) d \eta^{\prime},
\end{aligned}
$$

where $r=2\left|y^{\prime}-z^{\prime}\right|, \sigma_{r}=\left\{\eta^{\prime} \in \mathbb{R}^{2}:\left|y^{\prime}-\eta^{\prime}\right| \leq 2 r\right\}$. Using (3.25) and the inequality

$$
\left|\int_{\mathbb{R}^{2} \backslash \sigma_{r}} \mathbf{E}^{*}\left(y^{\prime}, \eta^{\prime}\right) d \eta^{\prime}\right| \leq c \delta
$$


which is a consequence of the Stokes formula

$$
\int_{\mathbb{R}^{2} \backslash \sigma_{r}} \mathbf{E}^{*}\left(y^{\prime}, \eta^{\prime}\right) d \eta^{\prime}=\int_{\ell} E(y-\eta) d l_{3}-\int_{\partial \sigma_{r}} E\left(y^{\prime}-\eta^{\prime}\right) d l_{3},
$$

$\ell=\left\{\eta^{\prime} \in \partial \sigma_{r}, \eta_{3}=\Phi_{k}^{*}\left(\eta^{\prime}\right)\right\}$, we obtain

$$
\left|\boldsymbol{u}\left(y^{\prime}\right)-\boldsymbol{u}\left(\eta^{\prime}\right)\right| \leq c \delta r^{\alpha^{\prime}}\|f\|_{\dot{C}^{\alpha^{\prime}}\left(\mathbb{R}^{2}\right)} .
$$

This completes the proof of (3.27).

Inequalities (3.28) are proved by similar arguments. If $v\left(y^{\prime}, t\right)=\left(\mathfrak{G}_{k}^{*}-\mathfrak{G}_{k}\right) \boldsymbol{f}$, then

$$
\begin{aligned}
v\left(y^{\prime}, t\right) & =\int_{0}^{t} d \tau \int_{\mathcal{U}_{k}(4 \delta)} \mathbf{G}^{*}\left(y^{\prime}-\eta^{\prime}, t-\tau\right) \cdot\left(\boldsymbol{f}\left(\eta^{\prime}, \tau\right)-\boldsymbol{f}\left(y^{\prime}, \tau\right)\right) d \eta^{\prime}, \\
\left|v\left(y^{\prime}, t\right)\right| \leq c \delta\|\boldsymbol{f}\|_{\dot{C}^{\alpha, 0}\left(\mathbb{R}_{T}^{2}\right)} & \\
v\left(y^{\prime}, t\right)-v\left(z^{\prime}, t\right) & =\int_{0}^{t} d \tau \int_{\sigma_{r}} \mathbf{G}^{*}\left(y^{\prime}, \eta^{\prime}, t-\tau\right) \cdot\left(\boldsymbol{f}\left(\eta^{\prime}, \tau\right)-\boldsymbol{f}\left(y^{\prime}, \tau\right)\right) d \eta^{\prime} \\
& -\int_{0}^{t} d \tau \int_{\sigma_{r}} \mathbf{G}^{*}\left(z^{\prime}, \eta^{\prime}, t-\tau\right) \cdot\left(\boldsymbol{f}\left(\eta^{\prime}, \tau\right)-\boldsymbol{f}\left(z^{\prime}, \tau\right)\right) d \eta^{\prime} \\
& +\int_{0}^{t} \int_{\mathbb{R}^{2} \backslash \sigma_{r}}\left(\mathbf{G}^{*}\left(y^{\prime}, \eta^{\prime}, t-\tau\right)-\mathbf{G}^{*}\left(z^{\prime}, \eta^{\prime}, t-\tau\right)\right) \cdot\left(\boldsymbol{f}\left(\eta^{\prime}, \tau\right)-\boldsymbol{f}\left(z^{\prime}, \tau\right)\right) d \eta^{\prime}, \\
\left|v\left(y^{\prime}, t\right)-v\left(\eta^{\prime}, t\right)\right| & \leq c \delta r^{\alpha^{\prime}}\|\boldsymbol{f}\|_{\dot{C}^{\alpha^{\prime}, 0}\left(\mathbb{R}_{t}^{2}\right)}, \quad t \leq T .
\end{aligned}
$$

Finally, we get

$$
\left|v\left(y^{\prime}, t\right)\right| \leq c \delta \int_{0}^{t}\|\boldsymbol{f}\|_{C^{\alpha^{\prime}}\left(\mathbb{R}^{2}\right)} d \tau \int_{\mathbb{R}^{2}} \frac{\left|\eta^{\prime}-y^{\prime}\right|^{\alpha^{\prime}} d \eta^{\prime}}{\left(\left|y^{\prime}-\eta^{\prime}\right|^{2}+(t-\tau)^{2}\right.} \leq c \delta \int_{0}^{t}\|\boldsymbol{f}\|_{C^{\alpha^{\prime}}\left(\mathbb{R}^{2}\right)} \frac{d \tau}{(t-\tau)^{1-\alpha^{\prime} / 2}}
$$

The proposition is proved.

To complete the proof of Theorem 2, we must estimate $\left\|\mathfrak{T}^{(4)} g\right\|_{C^{\alpha^{\prime}, 0}\left(S_{T}\right)}$. We have

$$
\left\|\varphi_{m} \mathfrak{T}^{(4)} g\right\|_{C^{\alpha^{\prime}, 0}\left(U_{m, t}\right)} \leq c \max _{k}\left(\left\|\widetilde{\mathfrak{T}}_{k}^{(4)} g_{k}\right\|_{\dot{C}^{\alpha^{\prime}, 0}\left(U_{k, t}\right)}+c \delta^{-\alpha^{\prime}} \sup _{U_{k, t}}\left|\widetilde{\mathfrak{T}}_{k}^{(4)} g_{k}\right|\right)
$$

where

$$
\tilde{\mathfrak{T}}_{k}^{(4)} g_{k}=\left(\left(\mathfrak{G}_{k}^{*}-\mathfrak{G}_{k}\right)\left(\mathfrak{S}_{k}^{*}-\mathfrak{S}_{k}\right)+\left(\mathfrak{G}_{k}^{*}-\mathfrak{G}_{k}\right) \mathfrak{S}_{k}+\mathfrak{G}_{k}\left(\mathfrak{S}_{k}^{*}-\mathfrak{S}_{k}\right)\right) \psi_{k} \mathfrak{R}_{k} g_{k}
$$

By Proposition 7,

$$
\begin{aligned}
\left\|\widetilde{\mathfrak{T}}_{k}^{(4)} g_{k}\right\|_{\dot{C}^{\alpha^{\prime}, 0}\left(U_{k, t}\right)} & \leq c \delta\left\|\psi_{k} \mathfrak{R}_{k} g_{k}\right\|_{C^{\alpha^{\prime}, 0}\left(U_{k, t}\right)} \\
& \leq c \delta\left(\left\|g_{k}\right\|_{C^{\alpha, 0}\left(U_{k, t}\right)}+\delta^{-\alpha^{\prime}} \sup _{U_{k, t}}\left|\mathfrak{L}_{k} g_{k}\right|\right),
\end{aligned}
$$

where $\mathfrak{L}_{k}$ is the integral operator with the kernel $L$ on $\mathbb{T}_{k}$ (see (3.4)). By (1.19),

$$
\mathfrak{L}_{k} g_{k}=\int_{0}^{t} d \tau \int_{\mathbb{R}^{2}} L\left(x^{\prime}-y^{\prime}, t-\tau\right)\left(g_{k}\left(y^{\prime}, \tau\right)-g_{k}\left(x^{\prime}, \tau\right)\right) d y^{\prime} .
$$

Hence, the last term in (3.30) is controlled by

$$
c \delta^{1-\alpha^{\prime}} \int_{0}^{t}\left\|g_{k}\right\|_{C^{\alpha^{\prime}, 0}\left(U_{k, \tau)}\right.} \frac{d \tau}{(t-\tau)^{1-\alpha^{\prime} / 2}},
$$

and $\mathfrak{T}^{(4)} g$ satisfies (3.9). This completes the proof of Theorem 2 . 


\section{ACKNOWLEDGMEnts}

The authors are thankful to Professor G. Grubb for a useful discussion and to Dr. E. V. Frolova for her valuable suggestions concerning the presentation of the material in this paper.

\section{REFERENCES}

[1] O. A. Ladyzhenskaya and V. A. Solonnikov, Solution of some non-stationary problems of magnetohydrodynamics for a viscous incompressible fluid, Tr. Mat. Inst. Steklov 59 (1960), 115-173. (Russian) $\operatorname{MR} 0170130(30: 371)$

[2] _ On linearization principle and invariant manifolds for problems of magnetohydrodynamics, Zap. Nauchn. Sem. Leningrad. Otdel. Mat. Inst. Steklov. (LOMI) 38 (1973), 46-93. (Russian) MR0377310 (51:13482)

[3] S. Mosconi and V. A. Solonnikov, On a problem of magnetohydrodynamics in a multi-connected domain, Nonlinear Anal. 74 (2011), no. 2, 462-478. MR2733223 (2011g:35320)

[4] Sh. Sahaev, Solution of the first initial-bondary value problem for nonstationary systems of Maxwell's equations (potential theory). I, Akad. Nauk Kazah. SSR Tr. Inst. Mat. i Meh. 2 (1971), 69-77. (Russian) MR0454424(56:12675)

[5] G. Grubb, Functional calculus of pseudodifferential boundary problems, Progr. Math., vol. 65, Birkhäuser, Boston, MA, 1996. MR1385196 (96m:35001)

[6] V. A. Solonnikov, An initial-boundary value problem for a generalized system of Stokes equations in a half-space, Zap. Nauchn. Sem. S.-Petgerburg. Otdel. Mat. Inst. Steklov. (POMI) 271 (2000), 224275; English transl., J. Math. Sci. (N. Y.) 115 (2003), no. 6, 2832-2861. MR1810619 (2002b:76045)

[7] V. I. Smirnov, A course of higher mathematics. Vol. IV, Gosudarstv. Izd. Tehn.-Teor. Lit., Moscow, 1951; English transl., Pergamon Press, Oxford, 1964. MR0177069 (31:1333)

[8] _ A course of higher mathematics. Vol. II, Nauka, Moscow, 1974; English transl., Pergamon Press, Oxford, 1964. MR0182688(32:171) MR0182688 (32:173)

St. Petersburg Branch, Steklov Mathematical Institute, Russian Academy of Sciences, Fontanka 27, 191023 St. Petersburg, Russia

E-mail address: Sahaev@pdmi.ras.ru

St. Petersburg Branch, Steklov Mathematical Institute, Russian Academy of Sciences, Fontanka 27, 191023 St. Petersburg, Russia

E-mail address: solonnik@pdmi.ras.ru

Received 4/AUG/2014

Originally published in English 